\title{
Characterizing Sources of Variability in Zebrafish Embryo Screening Protocols
}

\author{
Jon T. Hamm 1, Patricia Ceger 1, David Allen 1, Matt Stout 2, Elizabeth A. Maull 3, Greg Baker 4, \\ Amy Zmarowski 4, Stephanie Padilla 5, Edward Perkins 6, Antonio Planchart 7, Donald Stedman 8, Tamara Tal 5, \\ Robert L. Tanguay ${ }^{9}$, David C. Volz ${ }^{10}$, Mitch S. Wilbanks ${ }^{6}$ and Nigel J. Walker ${ }^{2}$ \\ ${ }^{1}$ Integrated Laboratory Systems, Research Triangle Park, NC, USA; ${ }^{2}$ Division of the National Toxicology Program, National Institute of Environmental \\ Health Sciences, National Institutes of Health, Research Triangle Park, NC, USA; ${ }^{3}$ National Toxicology Program Interagency Center for the Evaluation \\ of Alternative Toxicological Methods, Division of the National Toxicology Program, National Institute of Environmental Health Sciences, National \\ Institutes of Health, Research Triangle Park, NC, USA; ${ }^{4}$ Battelle, Life Sciences Research, Columbus, OH, USA; ${ }^{5}$ Integrated Systems Toxicology Division, \\ National Health and Environmental Effects Research Laboratory, Office of Research and Development, U.S. Environmental Protection Agency, Research \\ Triangle Park, NC, USA; ${ }^{6}$ United States Army Engineer Research and Development Center, Vicksburg, MS, USA; ${ }^{7}$ Department of Biological Sciences \\ and Center for Human Health and the Environment, North Carolina State University, Raleigh, NC, USA; ${ }^{8}$ Pfizer Pharmaceuticals, New London/Norwich, \\ CT, USA; ${ }^{9}$ Department of Environmental \& Molecular Toxicology, Oregon State University, Corvallis, OR, USA; ${ }^{10}$ Department of Environmental \\ Sciences, University of California, Riverside, CA, USA
}

\begin{abstract}
There is a need for fast, efficient, and cost-effective hazard identification and characterization of chemical hazards. This need is generating increased interest in the use of zebrafish embryos as both a screening tool and an alternative to mammalian test methods. A Collaborative Workshop on Aquatic Models and $21^{\text {st }}$ Century Toxicology identified the lack of appropriate and consistent testing protocols as a challenge to the broader application of the zebrafish embryo model. The National Toxicology Program established the Systematic Evaluation of the Application of Zebrafish in Toxicology (SEAZIT) initiative to address the lack of consistent testing guidelines and identify sources of variability for zebrafish-based assays. This report summarizes initial SEAZIT information-gathering efforts. Investigators in academic, government, and industry laboratories that routinely use zebrafish embryos for chemical toxicity testing were asked about their husbandry practices and standard protocols. Information was collected about protocol components including zebrafish strains, feed, system water, disease surveillance, embryo exposure conditions, and endpoints. Literature was reviewed to assess issues raised by the investigators. Interviews revealed substantial variability across design parameters, data collected, and analysis procedures. The presence of the chorion and renewal of exposure medium (static versus static-renewal) were identified as design parameters that could potentially influence study outcomes and should be investigated further with studies to determine chemical uptake from treatment solution into embryos. The information gathered in this effort provides a basis for future SEAZIT activities to promote more consistent practices among researchers using zebrafish embryos for toxicity evaluation.
\end{abstract}

\section{Introduction}

Traditional mammalian toxicity tests are time-intensive, expensive, and require both large amounts of test chemical and large numbers of animals (NRC, 2007; Rovida and Hartung, 2009). The cost and time needed to conduct these tests may limit the toxicity data available for the thousands of chemicals in commer- cial use. The need for toxicity information on these chemicals is driving interest in adopting test methods with higher throughput (Tice et al., 2013; Bugel et al., 2014).

In response to a number of factors, including the National Research Council report Toxicity Testing in the $21^{\text {st }}$ Century (NRC, 2007), testing initiatives such as the U.S. government's interagency Tox21 project (Attene-Ramos et al., 2013; Tice et al.,

Disclaimer: This document may not necessarily reflect official policy of any U.S. government agency. This manuscript has been subjected to review by the National Health and Environmental Effects Research Laboratory of the U.S. Environmental Protection Agency and approved for publication. Approval does not signify that the contents reflect the views of the Agency, nor does mention of trade names or commercial products constitute endorsement or recommendation for use.

Received April 16, 2018; Accepted October 30, 2018 Epub November 10, 2018; @ The Authors, 2018

ALTEX 36(1), 103-120. doi:10.14573/altex.1804162

Correspondence: Jon Hamm, PhD, ILS, P.O. Box 13501 Research Triangle Park, NC 27709 USA

(hammjt@niehs.nih.gov)
This is an Open Access article distributed under the terms of the Creative Commons Attribution 4.0 International license (http://creativecommons.org/licenses/by/4.0/) which permits unrestricted use, distribution and reproduction in any medium, provided the original work is appropriately cited. 
2013) and the U.S. Environmental Protection Agency's (EPA) ToxCast ${ }^{\mathrm{TM}}$ program (Dix et al., 2007) were developed to fundamentally shift current approaches used for hazard identification and characterization. New approaches include the use of high-throughput, cell-based screens to prioritize chemicals for further targeted toxicological testing. However, additional medium- to high-throughput models are needed to provide increased physiological relevance as well as to link in vitro observations to molecular, cellular, or physiological effects in whole animals (NRC, 2017).

Zebrafish (Danio rerio), which are being developed as physiologically relevant model organisms for medium throughput assays, are a small tropical fish native to the southeastern Himalayan region of Asia and are routinely maintained and bred in a laboratory setting (Lawrence, 2007; Lawrence and Mason, 2012; Dai et al., 2014). Zebrafish have relatively high fertility rates, rapid development with short intergenerational time, and a well-annotated genome with $\sim 70 \%$ concordance with mammalian species (Postlethwait et al., 2000; Howe et al., 2013). For these reasons, zebrafish have been used extensively in several fields, including environmental health science (Lieschke and Currie, 2007; Perkins et al., 2013; Aguirre-Martínez et al., 2017; Horie et al., 2017), ecotoxicology (Martins et al., 2007; Almond and Trombetta, 2016; de Oliveira et al., 2016), developmental biology (Solnica-Krezel et al., 1995; Lele and Krone, 1996; Elkouby, 2017), and genetics (Kinth et al., 2013; Varshney et al., 2015; White, 2015; Ceol and Houvras, 2016). Kinth et al. (2013) provides a recent review of literature for use of zebrafish as a model organism, including prominent areas of research, researchers, and research facilities.

Experimental throughput with adult zebrafish is an order of magnitude higher than that of mammals (Collins et al., 2008; Truong et al., 2016). There are, however, ethical concerns with the use of adult zebrafish (Braithwaite and Boulcott, 2007; Sneddon, 2009), and throughput of experiments using adult zebrafish remains below what is needed to screen vast numbers of chemicals. In response to these concerns and in an attempt to reduce, refine, or replace animal use (Russell and Burch, 1992; Tannenbaum and Bennett, 2015), the zebrafish embryo, which is considered to experience little or no pain, suffering, or distress (Strahle et al., 2012) has been investigated as a humane replacement for adult fish (Embry et al., 2010), and adopted for certain acute toxicity testing applications (OECD, 2013b). In the U.S., the National Institutes of Health Office of Laboratory Animal Welfare has determined that Public Health Service's Policy on Humane Care and Use of Laboratory Animals only applies to zebrafish after hatching (Bartlett and Silk, 2016). Similarly, as reviewed in Sneddon et al. (2017), Directive 2010/63/EU on the protection of animals used for scientific purposes specifies that fish become a protected animal once they are capable of independent feeding, or approximately 120 hours post-fertilization at $28^{\circ} \mathrm{C}$. Thus, the use of zebrafish embryos and larvae is in accordance with the $3 \mathrm{R}$ principles because it is considered an alternative model in embryonic stages (Ducharme et al., 2015).

The ability to generate thousands of developmentally synchronized embryos per day represents a significant advantage of zebrafish over mammals for testing purposes (Westerfield, 2000; Adatto et al., 2011). The small size of zebrafish embryos, approximately 1 to $1.5 \mathrm{~mm}$ in diameter (Kimmel et al., 1995; Forbes et al., 2010), makes them easy to maintain and treat in the multiwell plates that are standard for medium- to high-throughput platforms (Yozzo et al., 2013; Leet et al., 2014; Raftery et al., 2014; Vliet et al., 2017). Both the zebrafish embryo and the chorion, the outermost membrane surrounding the zebrafish embryo, are transparent, allowing for direct microscopic observation and evaluation throughout the entire developmental process (Hisaoka, 1958; Hisaoka and Battle, 1958). Additionally, the stages and timing of this process have been thoroughly documented (Kimmel et al., 1995; Westerfield, 2000; Nagel, 2002). The zebrafish embryo shares many characteristics with embryos of other vertebrates. Although zebrafish embryos lack some toxicologically relevant organs such as mammary glands, lungs (Perry et al., 2001), and a prostate gland (Van Slyke et al., 2014), they do possess other toxicologically relevant organ systems such as a liver that expresses cytochrome P450s (Tao and Peng, 2009; Otte et al., 2010; Weigt et al., 2011; Goldstone and Stegeman, 2012) and other metabolic enzymes (Kurogi et al., 2013; Klüver et al., 2014). These characteristics have made the zebrafish embryo an increasingly popular model for developmental toxicology (Dodd et al., 2000; Gunnarsson et al., 2008; Augustine-Rauch et al., 2010, 2016), and tests using zebrafish embryos are included in the battery of assays used in ToxCast ${ }^{\mathrm{TM}}$ (Braunbeck et al., 2005; Padilla et al., 2012a; Truong et al., 2014).

Several different groups have been working to validate zebrafish assays or harmonize zebrafish assay protocols for a number of purposes. Early efforts by the Organisation for Economic Co-operation and Development (OECD) to standardize fish assay protocols included adoption of test guidelines for the fish acute toxicity test (OECD, 1992), the early life stage toxicity test (OECD, 2013a), and the short-term toxicity test on embryo and sac-fry stages (OECD, 1998). These test guidelines allowed for the use of several different fish species including zebrafish. More recently, the fish embryo acute toxicity (FET) test, which assesses acute toxicity in zebrafish embryos up to $96 \mathrm{~h}$ post-fertilization (hpf), was approved as OECD Test Guideline 236 (OECD, 2013b; Busquet et al., 2014). One of the groups (Nagel et al., 1991; Schulte, 1994; Nagel, 2002) also worked on developing the zebrafish-specific version of TG 203 (Fish Acute Toxicity Test (OECD, 1992)), known as the DarT. This test was further refined by the addition of rat liver microsomes to the test system to allow for the evaluation of proteratogens (Busquet et al., 2008; Weigt et al., 2010). In 2014, a version of the DarT without

\footnotetext{
Abbreviations

DarT, 48-h embryo test with the zebrafish Danio rerio; DMSO, dimethyl sulfoxide; dpf, days post-fertilization; EPA, U.S. Environmental Protection Agency; FET, fish embryo acute toxicity; hpf, hours post-fertilization; IGG, SEAZIT information gathering group; logP, the affinity of a chemical for either aqueous and lipophilic solvents; NICEATM, NTP Interagency Center for the Evaluation of Alternative Toxicological Methods; NTP, National Toxicology Program; OECD, Organisation for Economic Co-operation and Development; SEAZIT, NTP Systematic Evaluation of the Application of Zebrafish in Toxicology; Tu, Tübingen zebrafish strain; ZET, zebrafish embryo teratogenicity
} 
microsomes was expanded from glass vials into 24-well tissue culture plates and tested in an OECD intra- and inter-laboratory evaluation (Busquet et al., 2014).

In 2010, Brannen et al. developed the zebrafish embryo teratogenicity (ZET) assay (Brannen et al., 2010), which used 24-well plates, dechorionated eggs, and embryos exposed statically from 4-6 hpf up to 5 days post-fertilization (dpf). This model was tested using 34 chemicals with in vivo rodent data on developmental toxicity. The model correctly categorized $87 \%$ of the chemicals (Brannen et al., 2010) as teratogenic or non-teratogenic. The ZET was further improved in 2010 by the establishment of more standardized morphological scores (Panzica-Kelly et al., 2010).

A consortium of drug development companies developed a basic protocol for the ZET assay; this protocol was then used to test a set of 20 chemicals in four laboratories to evaluate how specific protocol parameters affected assay performance (Gustafson et al., 2012). At the onset of the study two of the laboratories performed chemical uptake studies and found that for the chemicals used in the study, the presence or absence of the chorion did not affect chemical uptake. In these two laboratories, the ZET assay protocol was modified to include the chorion (Gustafson et al., 2012). Conversely, one laboratory tested the optimized protocol at the conclusion of Phase I and concluded that dechorionation slightly improved concordance with mouse, rat, and rabbit teratogenicity reference data, but required a complicated assay set-up. Phase II of this effort tested 38 chemicals in two laboratories (Ball et al., 2014). One laboratory used pond-derived fish, while the other used laboratory-bred fish. Both laboratories measured chemical uptake and found that chemicals with low $(<5 \%)$ uptake were toxic if sufficiently high $(1000 \mu \mathrm{M})$ concentrations of chemical were applied. Strain differences were not reported as a factor affecting concordance between the two laboratories. In 2015, Panzica-Kelly et al. (2015) re-evaluated the optimized protocol used in the Gustafson and Ball studies using dechorionated embryos as well as embryos with intact chorions and chorions weakened by enzymatic treatment. The embryos with weakened chorions or no chorion exhibited a slight (4\%) increase in chemical sensitivity compared to embryos with intact chorions.

As discussed in Beekhuijzen et al. (2015) and Planchart et al. (2016), the methodology for toxicity tests employing zebrafish embryos varies greatly between laboratories, with differences in the strain of fish used, timing and frequency of exposure, status of the chorion, exposure apparatus, endpoints measured, and scoring of phenotypic alterations. Unfortunately, the pharmaceutical consortium's studies left many unanswered questions regarding key parameters in the ZET assay protocol. Importantly, the studies did not examine the influence of exposure frequency on toxicity.

The pharmaceutical consortium's studies also did not resolve important questions surrounding the presence of the chorion. For example, the uptake studies were generated almost exclusively from pharmaceuticals (17/20 chemicals), raising the question of whether the results are valid for other types of chemicals. The fact that the detailed uptake data are not published (Gustafson et al., 2012) makes this question impossible to resolve. Panzica-Kelly et al. (2015) assert that while chorion removal or treatment slightly increased sensitivity, the complexity and set-up time added by this step would be prohibitive for most applications. This review also noted that at least two chemicals identified as teratogens in chorionated embryos were classified as non-teratogens when the chorion was removed or weakened, further complicating an interpretation of the role of the chorion in influencing uptake and toxicity (Panzica-Kelly et al., 2015).

Recognizing both the potential benefits and challenges of using zebrafish and other aquatic models in chemical screening applications, a group of U.S.-based academic and government scientists convened a workshop to identify and propose research initiatives to address the challenges. Among those participating were the laboratories generating data for the ToxCast ${ }^{\mathrm{TM}}$ screening effort and several additional laboratories using zebrafish as a model organism. The 2014 Collaborative Workshop on Aquatic Models and $21^{\text {st }}$ Century Toxicology was organized by the National Toxicology Program (NTP) Interagency Center for the Evaluation of Alternative Toxicological Methods (NICEATM), North Carolina State University's Center for Human Health and the Environment, Duke University, EPA, and the U.S. Food and Drug Administration. The purposes of the workshop were to explore and discuss how aquatic models could be used to screen and prioritize chemicals for in vivo testing and to assess mechanisms of chemical toxicity to human and environmental health. Discussions focused on how the techniques and knowledge of broad-based, interdisciplinary research could leverage the application of aquatic models to the field of environmental health (Planchart et al., 2016). Workshop participants identified the lack of standardized protocols as an impediment to broader acceptance of these models, and recommended that development of standardized protocols, validation, and subsequent regulatory acceptance would facilitate greater usage.

To address the need for standardized and validated protocols, in 2015 staff from NTP and NICEATM initiated the Systematic Evaluation of the Application of Zebrafish in Toxicology (SEAZIT) to gather the information and data required to achieve a higher degree of standardization for zebrafish embryo protocols. This work would in turn provide a scientific foundation for making decisions on the potential use of zebrafish embryos in chemical safety screening. SEAZIT's goals include gathering input from zebrafish investigators on protocol elements that are potential sources of outcome variability and facilitating a multi-laboratory evaluation of identified protocol variables. This report summarizes the findings of the initial phase of SEAZIT information gathering efforts and provides the basis for future activities to explore the potential utility of zebrafish embryo tests in toxicity evaluation.

\section{Methods and data sources}

The initial SEAZIT information gathering efforts focused on scrutinizing current screening methods and determining why investigators using zebrafish embryos in toxicity testing chose their specific experimental protocols. The rationale for this focus was that, given prior experiences described in the Introduction, it seemed appropriate to assess current best practices before proposing new harmonized protocols. 
To accomplish this, SEAZIT contacted investigators currently using zebrafish embryos for toxicity testing to assess their willingness to participate in group discussions as an information gathering group (IGG). The eight scientists were selected to include the laboratories generating data in zebrafish embryos for the ToxCast ${ }^{\mathrm{TM}}$ effort and to represent academia, industry, and government. The scientists ultimately selected to participate on the IGG represented six distinct laboratories including two from EPA and two from the U.S. Army (Tab. 1). SEAZIT team members developed a questionnaire to collect protocol component information from IGG members. The questionnaire is available as supplementary material. ${ }^{1}$ Some of the data collected included zebrafish strains, types of feed, preparation of system water, disease surveillance practices, embryo exposure conditions, and endpoints assessed. The questionnaire responses were transcribed, tabulated, and followed up by individual interviews to clarify details. Five group teleconferences were then held to discuss the group's findings. SEAZIT team members also reviewed literature from the participating laboratories and citations from PubMed that pertained to issues raised by the IGG.

\section{Results}

\subsection{Parameters to consider}

Despite the growing interest and use of zebrafish in scientific research (Lidster et al., 2017), and the publication of several reviews on general zebrafish care (Lawrence, 2011; Varga, 2011; Lawrence and Mason, 2012), reproductive biology (Lawrence, 2012), and health monitoring (Westerfield, 2000; Collymore et al., 2016), there is still considerable variation in zebrafish husbandry practices in animal facilities and research laboratories. The SEAZIT team discussions with the IGG suggest that husbandry practices and experimental protocol parameters fall into three distinct groups: those which the group felt were unlikely to affect study outcomes and those which we have termed "lesser" and "specific" parameters of concern. The IGG concluded that the lesser parameters of concern are those that theoretically could affect the outcomes of zebrafish experiments, but are not believed to be important sources of inter-laboratory variability. Conversely, variability in the specific parameters of concern could potentially nullify the outcomes and conclusions

Tab. 1: Information gathering group members

\begin{tabular}{|c|c|}
\hline Member & Affiliation \\
\hline Stephanie Padilla* & $\begin{array}{l}\text { Research Toxicologist } \\
\text { Integrated Systems Toxicology Division } \\
\text { National Health and Environmental Effects Research Laboratory } \\
\text { Office of Research and Development, EPA }\end{array}$ \\
\hline Edward Perkins & $\begin{array}{l}\text { Senior Scientist } \\
\text { Environmental Laboratory } \\
\text { U.S. Army Engineer Research and Development Center }\end{array}$ \\
\hline Antonio Planchart & $\begin{array}{l}\text { Assistant Professor } \\
\text { Department of Biological Sciences and Center for Human Health and the Environment } \\
\text { North Carolina State University }\end{array}$ \\
\hline Donald Stedman & $\begin{array}{l}\text { Senior Principal Scientist } \\
\text { Pfizer Pharmaceuticals }\end{array}$ \\
\hline Tamara Tal ${ }^{*}$ & $\begin{array}{l}\text { Biologist } \\
\text { Integrated Systems Toxicology Division } \\
\text { National Health and Environmental Effects Research Laboratory } \\
\text { Office of Research and Development, EPA }\end{array}$ \\
\hline Robert Tanguay & $\begin{array}{l}\text { Distinguished Professor } \\
\text { Department of Environmental \& Molecular Toxicology } \\
\text { Oregon State University }\end{array}$ \\
\hline David Volz & $\begin{array}{l}\text { Associate Professor } \\
\text { Department of Environmental Sciences } \\
\text { University of California, Riverside }\end{array}$ \\
\hline Mitch Wilbanks ** & $\begin{array}{l}\text { Research Biologist } \\
\text { Environmental Genomics and Systems Biology Team } \\
\text { U.S. Army Engineer Research and Development Center }\end{array}$ \\
\hline
\end{tabular}

*Stephanie Padilla and Tamara Tal utilize the same animal facility at EPA and many of their responses were combined to reflect the shared practices. ${ }^{* *}$ Mitch Wilbanks was interviewed and provided responses on laboratory procedures for the U.S. Army Engineer Research and Development Center.

\footnotetext{
1 doi:10.14573/altex.1804162s
} 
of embryo experiments. Both lesser and specific parameters of concern are discussed below.

\subsection{Lesser parameters of concern}

\subsubsection{Source and strain of fish used}

In early zebrafish research, fish were either wild-caught or obtained from commercial breeders or pet shops (Hisaoka, 1958; Hisaoka and Battle, 1958; Hisaoka and Firlit, 1960; Stanton, 1965). In the 1970s and 1980s, efforts to develop specific laboratory strains established the AB strain of zebrafish (Streisinger et al., 1981; Streisinger, 1984; Johnson and Zon, 1999). Investigators in Germany subsequently developed the Tübingen strain, which has been used extensively for the evaluation of mutations and genetic diversity (Haffter et al., 1996; Geisler et al., 2007; Brown et al., 2012). Many other strains and stocks of zebrafish have since been developed (summarized in Johnson and Zon, 1999).

It is unclear to what extent observed differences in zebrafish characteristics and behavior are due to genetics, as opposed to individual laboratory and husbandry practices (Ball et al., 2014). Strain-specific behavioral responses to various stimuli have been documented (Vignet et al., 2013; Gao et al., 2016; Quadros et al., 2016; Seguret et al., 2016; van den Bos et al., 2017), including differences in hearing sensitivity (Monroe et al., 2016), visual social preference (Barba-Escobedo and Gould, 2012), and growth performance in response to fasting (Meyer et al., 2013). To date, there is little evidence to indicate the existence of strain differences in sensitivity to toxicants (Chatla et al., 2016), or how genetic differences affect responses to toxicants (Coe et al., 2009). IGG members indicated that in the past, they have used or are using $\mathrm{AB}$, Tübingen, or other strains of zebrafish. However, a common theme in the IGG interviews was that fish used in toxicity studies should be as outbred as possible to avoid the effects caused by line inbreeding, as well as to differentiate zebrafish assays from mammalian-based toxicological screens, which use a very limited gene pool, making zebrafish data more relevant to the human and animal populations, which are genetically heterogeneous (Childs and Der Kaloustian, 1968; Brown et al., 2012; Zuberi and Lutz, 2016; Balik-Meisner et al., 2018a,b).

The zebrafish strains used by the IGG are listed in Table 2 . Several of the laboratories indicated that in the past they have switched strains to improve fecundity and embryo survival to the 5D strain, a strain originally derived from 5D Tropical (Plant City, FL USA), being a strain they have found to have high fecundity.

\subsubsection{Breeding}

All the laboratories reported differences in their breeding protocols, driven by individual research needs. The minimum breeding age reported varied both by laboratory and strain. Laboratories generally initiate breeding around four months of age and euthanize breeders at between nine and 24 months of age. Three laboratories mentioned that zebrafish are territorial (Spence and Smith, 2005; Spence, 2011) and that periodically placing previously isolated breeder fish into new groups seems to improve breeding success.

\subsubsection{Feed}

The adult and larval fish diets reported by laboratories at the time of interview are reported in Table 3. Following group discussion of current practices, several laboratories were investigating changing to the use of Gemma pellet diet (Skettring; Westbrook, $\mathrm{ME}$ ) without an additional supplementation of live foods to ensure greater definition of the diet. Feed information presented in the rest of this section will reflect the original IGG member reports.

IGG members obtain their fish food from several sources. All IGG members acknowledge that there is a potential for commercial fish foods to be a source of chemical contaminants (Maule et al., 2007; Berntssen et al., 2010; Nácher-Mestre et al., 2014). The majority of commercial suppliers of prepared diets used by IGG members do not provide information regarding pesticide and heavy metal content. One laboratory reported that their supplier tests for both pesticides and heavy metals, while two other laboratories test their diets in-house for mercury, cadmium, and

Tab. 2: Zebrafish strains used in participating laboratories

\begin{tabular}{|l|l|l|}
\hline Strain name & Source & IGG comment \\
\hline$A B$ & Aquatic BioSystems & \\
\hline$A B$ & ZIRC & Not very fecund \\
\hline Modified AB & In-house developed & Recently back-crossed to fish from ZIRC \\
\hline 5D & 5D Tropical & Selected for high fecundity \\
\hline fli1:egfp line (reporter gene strain) & Other laboratory & \\
\hline Tübingen & Not provided & \\
\hline Outbred wildtype strain & In-house developed & A mixture of several fish obtained from commercial and other \\
\hline Wildtype strain & laboratory sources (ZIRC) \\
\hline
\end{tabular}


Tab. 3: Adult and larval feeds used at participating laboratories as reported during the IGG interviews

\begin{tabular}{|l|l|l|l|}
\hline Laboratory & Prepared diet & Suppliers & Live food \\
\hline $1^{\text {a }}$ & $\begin{array}{l}\text { Zeigler Adult ZF Diet } \\
\text { Ziegler Larval ZF Diet }\end{array}$ & Zeigler Bros, Inc. (Gardner, PA, USA) & Artemia (adults) \\
\hline 2 and $3^{\text {b }}$ & GEMMA micro & $\begin{array}{l}\text { Skretting (Tooele, UT, USA) } \\
\text { Reed Mariculture Inc. (Campbell, CA, USA) }\end{array}$ & $\begin{array}{l}\text { Artemia (adults and larvae) } \\
\text { Rotifers (larvae) }\end{array}$ \\
\hline 4 & $\begin{array}{l}\text { In-house derived, described } \\
\text { in Miller et al. (2014) }\end{array}$ & NA & None \\
\hline 5 & Zeigler Adult ZF Diet & $\begin{array}{l}\text { Zeigler Bros, Inc. (Gardner, PA, USA) } \\
\text { Reed Mariculture Inc. (Campbell, CA, USA) }\end{array}$ & $\begin{array}{l}\text { Artemia (adults) } \\
\text { Rotifers (larvae) }\end{array}$ \\
\hline $6^{\text {c }}$ & Aquatox Flake & Zeigler Bros, Inc. (Gardner, PA, USA) & Artemia (adults and larvae) \\
\hline $7^{\text {d }}$ & $\begin{array}{l}\text { Othohime Pellet } \\
\text { Zeigler Larval AP 100 }\end{array}$ & $\begin{array}{l}\text { Reed Mariculture Inc. } \\
\text { (Campbell, CA, USA) } \\
\text { Pentair (Cary, NC, USA) }\end{array}$ & INVE Artemia (adults and larvae) \\
\hline
\end{tabular}

aLaboratory 1 now uses GEMMA Micro 75 as a larval diet and GEMMA Micro 300 as an adult diet and has discontinued the use of Artemia. ${ }^{b}$ Laboratories 2 and 3 share the same fish facility and use the same feed. ${ }^{\circ}$ Laboratory 6 is phasing out the Aquatox Flake and live food and is switching to GEMMA. ${ }^{\text {L Laboratory }} 7$ now uses Gemma Micro 75 for larvae up to $20 \mathrm{dpf}$, Gemma Micro 150 for juveniles up to $60 \mathrm{dpf}$, and Gemma 300 for adults, and no longer uses live food.

lead content. None of the laboratories had any information about pesticide residue content in feeds.

Five of the six laboratories supplement adult fish diets with brine shrimp (various Artemia species) or other live foods. These laboratories feed rotifers or Artemia nauplii to larval fish from hatching until approximately 10 days of age before transitioning to Artemia (Seale, 1933; Nash, 1973) and pellet or flake food. IGG members whose laboratories do not supplement adult fish diets with live foods mentioned concerns with the variability in nutrient content and the potential for live foods to be a source of pathogens, heavy metals, and/or pesticide contamination, all of which have been documented by other researchers using aquatic models (Chen and Lru, 1987; Cook et al., 2008; Vikas et al., 2012; Lu et al., 2013; Karlsen et al., 2015). In-house testing of Artemia eggs and adults at one IGG laboratory identified mercury present in both. Based on these concerns, that laboratory switched to exclusive use of commercial diets and no longer uses live foods in their fish diet (Miller et al., 2012).

\subsubsection{Water}

All laboratories use either well water or municipal water in their aquaria. Water is filtered through a reverse osmosis filter and then reconditioned by adding aquarium salt and adjusting the $\mathrm{pH}$ before or after it is added to aquaria sump systems. Water circulated within the aquaria systems is mechanically filtered to remove solid particulates, chemically filtered using activated charcoal, and sterilized using ultraviolet light. One laboratory also uses a fluidized sand biofilter prior to charcoal filtration. All laboratories maintain water temperature at approximately $28^{\circ} \mathrm{C}$ for adult fish and embryos, although two laboratories reduce water temperature to $26^{\circ} \mathrm{C}$ to slow embryonic development and allow for longer chemical exposures during critical periods of susceptibility. Water temperature is continuously monitored by all laboratories. Monitoring of other parameters such as $\mathrm{pH}$, conductivity, salinity, and ammonia content varied among the laboratories, with some continuously monitoring these parameters and others periodically spot-checking them in an unspecified percentage of experimental tanks using commercial aquarium test kits. All laboratories indicated that they performed daily partial water changes, with some laboratories having automated changes and others performing them manually. Water changes were the most commonly used method to remedy out-of-range water parameters. Water parameters are summarized in Table 4.

\section{Tab. 4: Water parameters in IGG laboratories}

\begin{tabular}{|l|l|}
\hline Parameter & Range \\
\hline Water temperature & 26.0 to $28.5^{\circ} \mathrm{C}$ \\
\hline $\mathrm{pH}$ & 7 to 8 \\
\hline Ammonia* & 0.001 to $0.8 \mathrm{ppm}$ \\
\hline Nitrites* $^{*}$ & 0 to $0.1 \mathrm{ppm}$ \\
\hline Nitrates $^{*}$ & 0 to $20 \mathrm{ppm}$ \\
\hline Chlorine $^{*}$ undetectable \\
\hline Salinity & $<1 \mathrm{ppm}$ \\
\hline Dissolved oxygen & $>4 \mathrm{ppm}$ \\
\hline Conductivity & 200 to $1248 \mu \mathrm{S}$ \\
\hline
\end{tabular}

Abbreviations: $\mathrm{C}=$ Celsius; $\mathrm{ppm}=$ parts per million; $\mathrm{ppt}=$ parts per thousand; $\mu \mathrm{S}=$ microsiemens

*Some laboratories did not measure ammonia, nitrites, or nitrates separately, but reported values as total nitrogen. 
Tab. 5: Common fish diseases observed at participating laboratories

\begin{tabular}{|l|l|}
\hline Disease name & Causative organism(s) or conditions \\
\hline Noninfectious nephrocalcinosis & $\begin{array}{l}\left.\text { High } \mathrm{CO}_{2} \text { (e.g., > } 12 \mathrm{mg} / \mathrm{l}\right) \text { in water or excessive levels of calcium and magnesium in the diet. } \\
\text { The use of calcium carbonate (rather than sodium bicarbonate) to buffer water in recirculating } \\
\text { systems has been associated with the condition. }\end{array}$ \\
\hline Gill epithelial hyperplasia & $\begin{array}{l}\text { A number of causes including protozoal, parasitic, and bacterial infection; also poor water } \\
\text { quality (high ammonia, nitrite, etc.). }\end{array}$ \\
\hline $\begin{array}{l}\text { Egg-associated inflammation and } \\
\text { fibroplasia }\end{array}$ & $\begin{array}{l}\text { Unclear, possibly abnormal egg retention and absorption; in some cases, may be due to } \\
\text { infection with Mycobacteria. }\end{array}$ \\
\hline Mycobacteriosis & Mycobacteria marinum, M. chelonae, M. fortuitum, and other species \\
\hline Piscinoodinium & Piscinoodinium pillulare \\
\hline Microsporidiosis & Pseudoloma neurophila \\
\hline Aerocystitis pseudoloma & Pseudoloma neurophilia \\
\hline
\end{tabular}

Tab. 6: Tissue culture plate size, typical media volume, and number of embryos per well

\begin{tabular}{|l|l|l|}
\hline Plate size & Volumes reported & Number of embryos \\
\hline T25 and T75 culture flask & $25-30 \mathrm{ml}$ & $10-50$ \\
\hline $1.5 \mathrm{ml}$ glass plates & $250 \mu \mathrm{l}$ & 1 \\
\hline 24 well plates & $1-2.5 \mathrm{ml}$ & $1-5$ \\
\hline 96 well plates & $0.25 \mathrm{ml}$ & 1 \\
\hline 384 well plates* & $0.05 \mathrm{ml}$ & 1 \\
\hline
\end{tabular}

* The laboratory that uses 384-well plates indicated that development of the embryo limits the length of the exposure period to $\sim 72 \mathrm{hpf}$.

Larvae are approximately $\sim 3 \mathrm{~mm}$ long and the size of the wells of a 384-well plate is insufficient to house larvae for a prolonged time.

\subsubsection{Disease and disease monitoring}

Five of the six laboratories perform routine disease monitoring; three of these use sentinel fish in this process. Diseases routinely monitored include mycobacteriosis, and microsporidiosis, as well as fresh water velvet disease arising from parasitism by $\mathrm{Pi}$ scinoodinium. Several common fish diseases (Matthews, 2004; Murray et al., 2011; Whipps et al., 2012) have been observed in the IGG facilities (Tab. 5). Disease diagnosis is performed by trained laboratory staff, and confirmed, if necessary, by a veterinary pathologist. Diseased fish are euthanized and the tanks these fish resided in closely monitored. Several IGG members use quarantine procedures for any newly acquired fish to prevent introduction of disease into breeding stocks, and they strongly recommended this practice be used for all laboratories.

\subsubsection{Embryo exposure conditions}

Exposure initiation time varies situationally within laboratories as well as across laboratories. The most common time to initiate exposure reported by IGG members is 5-6 hpf with a range from as early as $0.75 \mathrm{hpf}$ to as late as $24 \mathrm{hpf}$. Decisions about when to initiate and how long to expose embryos to the test chemical are often driven by the endpoint of interest. For example, exposure on or after $24 \mathrm{hpf}$ can increase the chance of finding true vascular disruption rather than vascular disruption secondary to gross morphological malformation (McCollum et al., 2016). One lab- oratory indicates that beginning exposure at 5-6 hpf allows for selection of embryos that appear to be developing normally, thus reducing background abnormalities. Another laboratory initiates exposure at $24 \mathrm{hpf}$ so that they can identify fish that fail to survive mechanical removal of the chorion.

While the FET test, which measures acute lethality (OECD, 2013b; Braunbeck et al., 2015) established a defined chemical exposure regime, the period of exposure for other toxicological applications varies. Exposure lengths reported by the IGG range from 4 to 144 hours, depending on the endpoints of interest.

Embryos are generally placed in multiwell plates for chemical exposure and evaluation. The size of plates used, volume of medium per plate, and the number of embryos per well are listed in Table 6. Representative images of zebrafish embryos in 384-well and 96-well tissue culture plates are provided in Figures 1 and 2. Two laboratories place the embryos in the multiwell plates into dark incubators; other laboratories, expressing concerns about circadian rhythms, house their embryos in a controlled light/dark environment, with 14 hours of light and 10 hours of darkness.

The medium used to grow the embryos varies. Three laboratories use E2 medium, with one of these supplementing the medium with methylene blue to inhibit fungal growth. Of the other three laboratories, two use E3 medium (NüssleinVolhard and Dahm, 2002), and one uses Hanks' Balanced Salt Solution. 

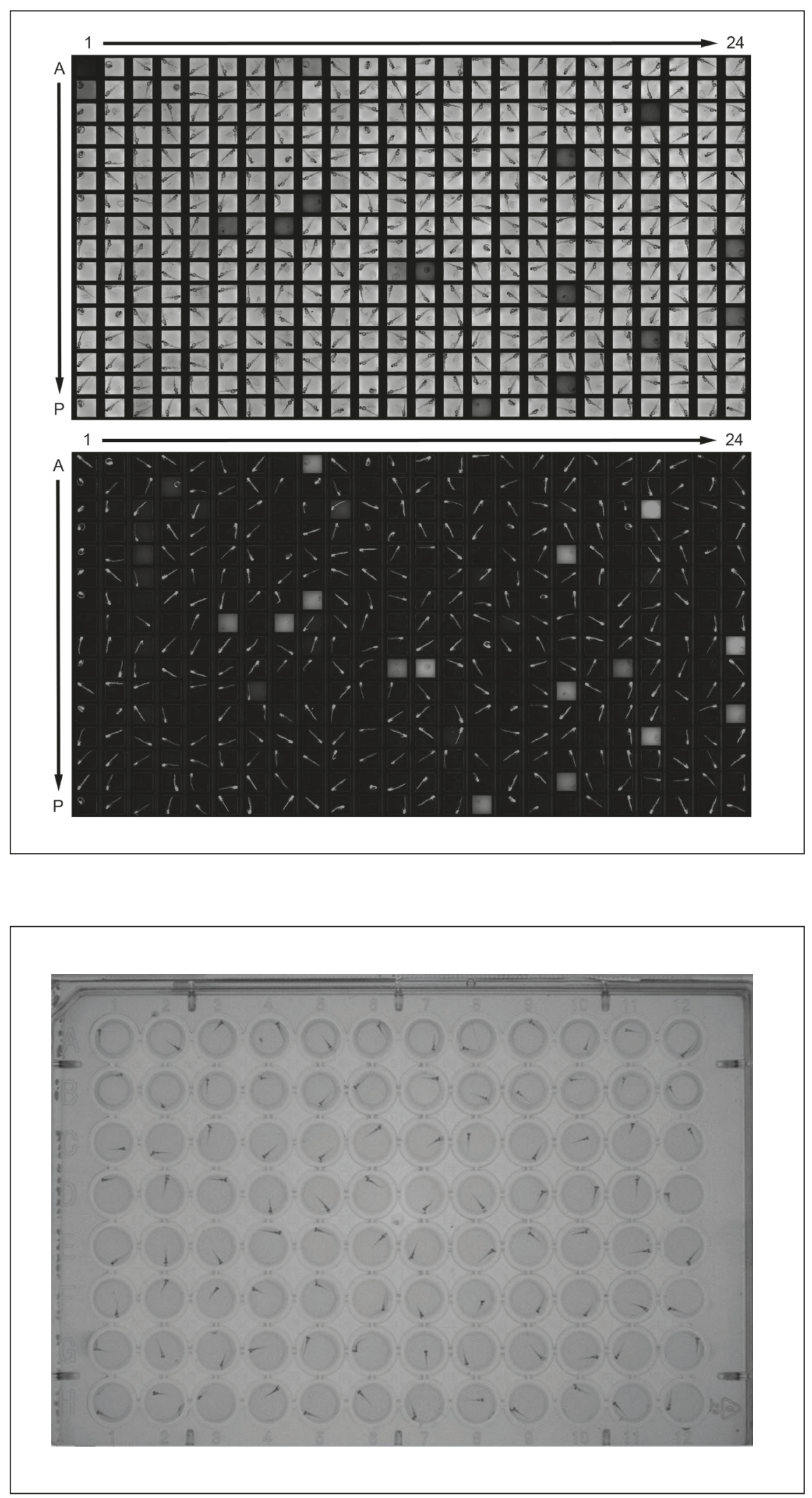

Fig. 1: Zebrafish embryos in a 384-well tissue culture plate

Photographs of transgenic fluorescent zebrafish embryos taken under a microscope using transmitted light in the top panel $(A)$ and using fluorescence capture in the bottom panel (B). Images are captured simultaneously. One zebrafish embryo is immersed in $50 \mu$ l of embryo medium in each $3 \times 3 \mathrm{~mm}$ well of the 384-well tissue culture plate. The embryos in this image were placed in the well at $5 \mathrm{hpf}$ and the image was taken at $72 \mathrm{hpf}$.

Fig. 2: Zebrafish embryos in a 96-well tissue culture plate

Photograph of zebrafish embryos in a 96-well tissue culture plate, taken under a microscope using transmitted light. 
Solvents used for chemical formulations included dimethyl sulfoxide (DMSO), water, and ethanol. DMSO, the most commonly used solvent, was used in final concentrations ranging from $0.1 \%$ to $1 \%$. There are concerns that DMSO may interfere with xenobiotic metabolism (David et al., 2012), alter locomotor activity in zebrafish larvae (Chen et al., 2011), and cause proteotoxic and embryotoxic effects (Hallare et al., 2006). To minimize these potential issues, laboratories prefer to keep DMSO concentrations to less than $1 \%$, and none have observed any behavioral or developmental effects at that level. Two laboratories mentioned concerns that test chemicals may change the $\mathrm{pH}$ of the exposure solution, which may alter the bioavailability of the chemical (Erickson et al., 2006; Armitage et al., 2017), or may potentially be fatal to the embryos exposed to $\mathrm{pH}$ extremes. The FET test prescribes that "the test solution should be in the range of 6.5 to 8.5 " and and further states that it "not vary within this range by more than 1.5 units during the course of the test. If the $\mathrm{pH}$ is not expected to remain in this range, then $\mathrm{pH}$ adjustment should be done prior to initiating the test. The $\mathrm{pH}$ adjustment should be made in such a way that the stock solution concentration is not changed to any significant extent and that no chemical reaction or precipitation of the test chemical is caused (OECD, 2013b)." Only one IGG laboratory stated that they measure and adjust the $\mathrm{pH}$ of dosing solutions and noted that they have found toxicity can be alleviated once $\mathrm{pH}$ was adjusted closer to neutral.

\subsubsection{Physio-chemical properties}

The factor universally agreed as impacting exposure was chemical solubility in zebrafish embryo medium. IGG members typically consider a chemical's $\log \mathrm{P}$, the affinity of a chemical for either aqueous and lipophilic solvents (usually octanol and water). This parameter is presumed to affect chemical uptake by adult zebrafish and embryos based on the assumption that highly water-soluble (i.e., hydrophilic) chemicals will stay in the water column and are unlikely to cross the lipid-rich cell membrane (Gustafson et al., 2012; Padilla et al., 2012b; de Koning et al., 2015; Ducharme et al., 2015) Conversely, lipophilic chemicals will more easily transverse cell membranes gaining entrance to the organism. The IGG investigators stated that extremely water-soluble chemicals are likely to produce false negative results due to little to no uptake, while insoluble chemicals cannot be tested in an aqueous exposure. One laboratory visually checks the wells of test plates under a microscope for precipitation from DMSO solutions as an indication of low solubility, and discontinues the experiment if it is observed. Another laboratory continues to conduct a test in the presence of chemical precipitation on the assumption that at least some of the chemical will partition into the culture medium and expose the zebrafish embryos; however, at that point it is extremely difficult to determine the exposure concentration. The IGG members agree that $\log \mathrm{P}$ influences uptake, but does not necessarily determine toxicity; however, they suggest that under repeat-dosing scenarios, logP appears to correlate with potency.

High volatility, a factor raised during the 2014 workshop as a physio-chemical property that could render a chemical unsuitable for testing, was not considered a limiting factor as all labo- ratories had contingency plans in place, e.g., sealing test plates for use with very volatile chemicals.

\subsubsection{Test plate controls and acceptability criteria}

All the IGG members indicate that they have experimental acceptance criteria based on fecundity and fertility. These criteria are evaluated before eggs are placed in the multiwell test plates. All laboratories monitor the number of eggs produced and fertilized per spawn. They also record the proportion of viable eggs, i.e., eggs that are transparent, with no sign of coagulation (OECD, 1998). All IGG members state that if any of these parameters are abnormal, the experiment is discarded. However, only one laboratory has a minimum acceptable viability level, which they set at $70 \%$.

All investigators stress the importance of producing as many fertilized, viable, "normal" eggs as possible. Furthermore, they indicate that reduced fecundity and/or egg quality is often the first sign of husbandry troubles in an aquaculture facility. One investigator notes that, in their laboratory's experience, the first parameter their laboratory staff check when egg production decreases among laboratory fish is the quantity and quality of the diet. Next, they investigate fish stressors such as water quality fluctuations or altered light/dark cycles. Such stressors can also negatively affect egg production and could eventually lead to illness and death.

Among the laboratories surveyed, use of positive controls and the chemicals used varies broadly. Two laboratories found after multiple years of testing that use of positive controls failed to add value to their assays, and thus discontinued using them. One laboratory uses different positive controls based on the chemical class of the study chemicals, e.g., 2,3,7,8-tetrachlorodibenzodioxin for chemicals that interact with the aryl hydrocarbon receptor. One laboratory uses chlorpyrifos at two different concentrations, one selected to be acutely toxic, and the other increasing malformations. One laboratory uses 3,4-dichloroaniline, the recommended control for the FET test (OECD, 2013b). This laboratory reports that the onset of exposure affected the toxicity of 3,4-dichloroaniline, with exposure beginning at $3 \mathrm{hpf}$ causing increased mortality, while exposures beginning at $6 \mathrm{hpf}$ cause increased malformations (Scheil et al., 2009). Finally, one laboratory reports that they routinely use either thiram or dithiocarbamate.

Regarding use of negative control chemicals, only one laboratory indicates they run simultaneous exposure of a negative control chemical with the choice of the negative control dependent on what they are evaluating. Another laboratory uses embryo rearing medium as the negative control, while the remaining laboratories utilize a solvent-only exposure as a negative control.

All participating laboratories use in-house test plate acceptability criteria that assess mortality, malformations, or both in solvent control-exposed embryos for each study. The percentage of live embryos required for an acceptable experiment varies between laboratories with the most common cutoff being $85 \%$.

\subsubsection{Endpoints and data collection}

The endpoints assessed, methods of data collection, and criteria used to evaluate endpoints vary among all laboratories. The most commonly collected endpoints include mortality, edema (peri- 
cardial, yolk sac, and other sites), skeletal malformations (including the spine and jaw), and a measure of total length, which are evaluated to some degree by all laboratories. Spontaneous tail movement at 24 hours and heartbeat are assessed by a number of laboratories, as are swim bladder inflation and hatching in laboratories that use chorionated embryos.

The interrelatedness of endpoints is an area of active investigation. One investigator mentioned that, in their laboratory's experience, swim bladder inflation, edema, and failure to thrive are all signs of generalized toxicity. These findings are sequential: the edema develops first, the swim bladder then fails to inflate, and ultimately the larva fails to thrive. Of note, a meta-analysis by Ducharme et al. (2013) found that altered hatching rate correlated with 20 other endpoints, including several gross morphology endpoints such as curvature of the spine, changes in size, and yolk sac edema, as well as several signaling pathway changes. Recently, Zhang et al. (2017) used a Bayesian method to analyze toxic responses to a large set of chemicals using 17 phenotypic alterations in zebrafish to quantify endpoint utility. Their results suggest that this approach improves identification of significant morphological effects and that a developmental cascade may be evaluated by analyzing the relationships among endpoints.

Approaches to data collection vary. The 2014 workshop featured several presentations of results obtained using automated image capture systems. IGG members report experience with several of these systems, including Array Scan (Cellomics, Inc., Pittsburgh, PA), Noldus System DanioVision with the DanioScope (Noldus Information Technology Inc. Leesburg, VA), VAST System (Union Biometrica, Holliston, MA), Molecular Devices ImageXpress (Molecular Devices, Sunnyvale, CA), and custom-built laboratory imaging systems. All of these systems use proprietary image collection and analysis software. One IGG member stated that their laboratory's evaluations require that the embryo be assessed from multiple orientations; at the time of these interviews, none of the available imaging systems allowed for this flexibility, so they are continuing to evaluate embryos manually. Other IGG members point out that several endpoints such as swim bladder inflation and head size cannot be measured by some of the automated systems. Some imaging systems require operators to manually orient agarose-embedded embryos before imaging, adding time and cost to the assay, as well as risking damage to the embryo during manipulation. Two participating laboratories use the VAST System, which addresses this issue by automatically loading zebrafish from reservoirs or multiwell plates and positioning and rotating them for imaging without damage to the embryo (Pardo-Martin et al., 2010).

\subsection{Specific parameters of concern}

\subsubsection{Static vs. static renewal}

The IGG investigators agreed that the method of exposing embryos to the test chemical is a key factor in experimental design. This parameter might reasonably be expected to influence the amount of chemical in the medium and in the embryo, and variability can affect the outcomes and conclusions of exposure experiments.
The three methods used to expose fish to test chemicals are static, static-renewal (i.e., semi-static), and flow-through (OECD, 2002). In the static method, the test chemical is added to the culture medium at the beginning of the experiment, and this exposure solution is used for the duration of the experiment without replacement of the culture medium or replenishment of the test chemical. The static-renewal method designates certain time points during the experiment at which the test solution is replaced with a fresh mixture of culture medium and test chemical; this is most typically done every 24 hours. The flow-through method requires a system in which test solution can be constantly circulated through the exposure environment, with monitoring and adjustment of test chemical concentration, simulating real-world aquatic exposure conditions.

Most zebrafish embryo chemical toxicity testing protocols use either the static or static-renewal exposure methods. As reviewed in Beekhuijzen et al. (2015), the choice of exposure method can be based on the physicochemical properties of the study chemicals or practical considerations of use in the laboratory. Static exposure protocols are easy and economical, as the test chemical does not need to be freshly measured out for each chemical exposure, and there is no additional time or labor required to replace the medium. Static-renewal protocols can help mitigate the loss of chemical due to volatilization, decomposition, or adherence to the exposure vessel while allowing for the use of multiwell test plates. Although Lammer et al. (2009) developed a flow-through setup using 24-well plates as test chambers, flow-through is generally incompatible with the use of tissue culture plates and can require significant amounts of test chemical.

One IGG member reported that in their laboratory's experience, the static-renewal method increased death and malformation rates in their controls due to manipulation of the embryos. Their (unpublished) evaluation of chemical uptake on a panel of over 100 chemicals found that, with a single exception, all chemicals were detected in the embryos to some extent, indicating that renewing test chemical was not necessary to achieve uptake.

Another investigator, whose laboratory uses 384-well test plates in its exposure protocol, indicated that renewal is not possible in their setup due to an inability to find pipette tips fine enough to fit into the wells and aspirate the medium without also inadvertently removing embryos. For that group, the advantages of using a 384-well plate platform (i.e., quadrupling experimental $\mathrm{N}$, using less test chemical, and more efficient high content time-lapsed imaging) outweigh any potential concerns about medium renewal.

Some IGG members reported that they only use the renewal method if significant metabolism of the test chemical by the zebrafish embryos is anticipated. One of the laboratories described a partial renewal protocol in which $40 \%$ of the medium in a well was replaced, thereby decreasing the chance of embryo desiccation by removing too much medium or accidentally disturbing the embryo with the pipette tip. This approach is required for dechorionated embryos, although with chorionated embryos, $100 \%$ of the medium can be replenished using mesh-bottom inserts to lift out the embryos, blot, and return to fresh exposure medium. 


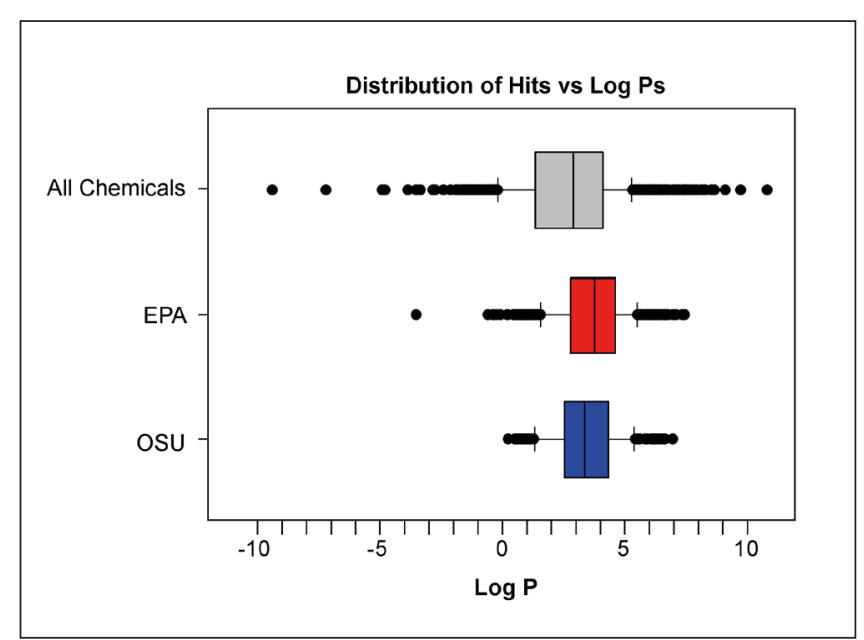

Fig. 3: Distribution of active chemicals at EPA and Oregon State University in comparison to logP

ToxCast ${ }^{\mathrm{TM}}$ chemicals that were determined to be active at both EPA and Oregon State University (OSU) were plotted based on their $\log \mathrm{P}$. The top box plot (grey box) is the logP distribution of all the chemicals in ToxCast ${ }^{\mathrm{TM}}$ Phase I and II. The middle box plot (red box) is the distribution of the actives reported by the EPA laboratory using chorionated embryos and semi-static dosing. The lower box (blue box) is the distribution of the actives reported by OSU using dechorionated embryos and static dosing. Results demonstrate that active chemicals at both laboratories share similar distribution of lipophilicity.

As a group, four of the IGG members reported that their laboratories exclusively use static exposures, while three of the laboratories use both static and static-renewal exposures. There was a difference of opinion about how the use of static versus static-renewal methods might influence toxicity. Some members stated that repeat exposure is capable of producing exceedingly high body burdens while others noted that false negative results are more likely in a single-static-exposure scenario when uptake is limited. IGG members agreed that a better understanding of toxicokinetics in zebrafish would help clarify the effect of different exposure methods on toxicity.

To date, a comparison of toxicity either within or between laboratories to a defined set of chemicals simultaneously exposed to zebrafish embryos via static and static renewal has not been published. The US EPA and Oregon State University laboratories, however, have tested many overlapping chemicals within the ToxCast ${ }^{\mathrm{TM}}$ effort. While the laboratories differ in a number of experimental design parameters, differences in the status of the chorion and exposure frequency are considered the most relevant to study outcome. Unpublished data from these laboratories suggests that regardless of design parameters, the laboratories identify biologically active chemicals within a similar chemical space (Fig. 3). However, repeated dosing appeared to be associated with a greater frequency of active chemicals (Fig. 4), a hypothesis that needs to be confirmed with controlled studies given the differences between the two laboratories regarding experimental design variables (unpublished data).

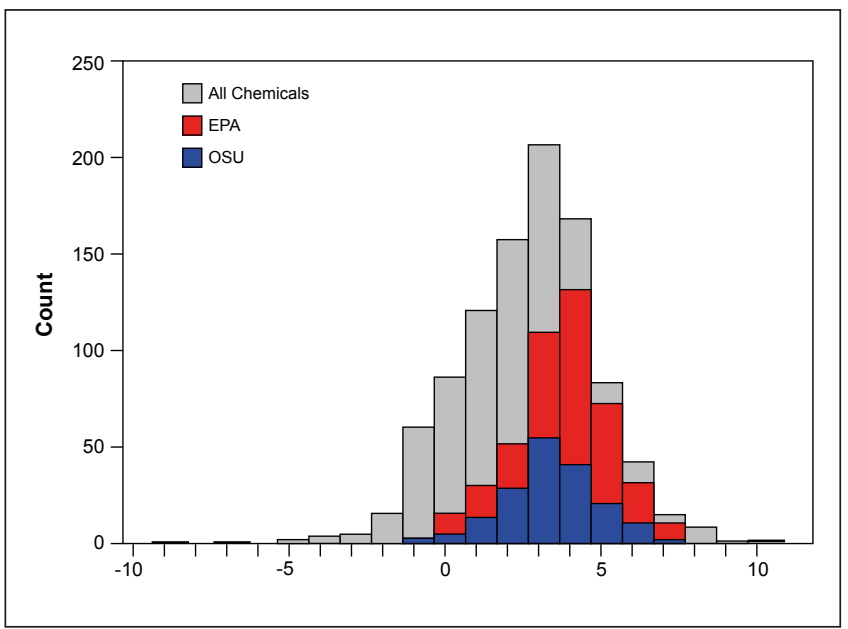

Fig. 4: Total numbers of active chemicals as determined by EPA and Oregon State University

All overlapping ToxCast ${ }^{\mathrm{TM}}$ chemicals run at both EPA and Oregon

State University (OSU) were plotted against logP along with the numbers of active chemicals at each institution. Results demonstrate that a greater number of chemicals were active when using chorionated embryos and semistatic dosing conditions (i.e., EPA protocol).

\subsubsection{Chorion status}

The general structure of zebrafish eggs is shown in Figure 5. The outermost membrane, the chorion, overlays the viscous fluid-filled perivitelline space, the vitelline membrane, and the blastoderm (or embryo) and yolk (Jones et al., 1978; Rawson et al., 2000). The chorion is an acellular envelope (Bonsignorio et al., 1996) about $3.5 \mu \mathrm{m}$ thick (Rawson et al., 2000). It is highly fenestrated with pores that are approximately $0.5 \mu \mathrm{m}$ in diameter (Hisaoka, 1958; Rawson et al., 2000), which have been shown to block the movement of molecules in excess of $3 \mathrm{kDa}$ molecular weight (Creton, 2004). In addition to molecule size, the ability of a chemical to pass through the chorion is also affected by its physicochemical properties (Kim and Tanguay, 2014), ionic charge (Cameron and Hunter, 1984; Finn, 2007), electrostatic properties (Burnison et al., 2006), and the concentration of DMSO used in the test system (Kais et al., 2013). For these reasons, some investigators use microinjection techniques to bypass the chorion (Janik et al., 2000) or mechanical, enzymatic, or automated robotic approaches to remove the chorion to ensure the embryos are exposed to test chemicals.

Four of the six IGG investigators use microinjection in their laboratory work, but none use it routinely to deliver test chemical to zebrafish embryos. Members indicated that they found microinjection to be an unacceptable source of uncertainty given that the volumes used are extremely small (nl). Additionally, there is a wide variation in the inner diameters of both handmade and commercially available microinjection needles, making it 
difficult to deliver consistent volumes of chemical to the embryos. The IGG members also expressed concern that injected chemicals may not enter the embryo, but instead might partition to the yolk or get trapped in the perivitelline space.

All IGG members indicated that they had tried either mechanical (Henn and Braunbeck, 2011) or enzymatic (Mandrell et al., 2012) chorion removal. Two laboratories reported that they dechorionate regularly. One laboratory indicated that if a comparison between exposed dechorionated and chorion-intact embryos detected exposure-related differences in their initial studies, dechorionated embryos were used in subsequent assays. One laboratory that uses chorion-intact embryos stated that removal of the chorion very rarely has any effect in their experience. Another laboratory stated that for exposure of nanomaterials, removal of the chorion can be critical.

IGG members from the four laboratories that used chorion-intact embryos routinely expressed a number of concerns:

- Dechorionation adds extra time and cost and reduces throughput.

- Dechorionated embryos are fragile and require either special equipment or training to avoid damaging the embryos when manipulating them.

- Dechorionated embryos stick to the nylon mesh plate inserts used in the IGG member's laboratory resulting in mortality.

- Dechorionation changes the embryos' orientation in the well and can interfere with image capture.

- An increase in background malformation and death is observed in dechorionated embryos (Henn and Braunbeck, 2011; Mandrell et al., 2012).

- Chorion removal prevents the use of hatching as an endpoint.

- Chorion removal is associated with altered behavior in embryonic, larval, and adult fish (Ninness et al., 2006; Thomas et al., 2009).

These investigators pointed out that the literature on the utility of dechorionation is divided, with some laboratories indicating that the chorion might be more permeable than previously believed (Wiegand et al., 2000; Gustafson et al., 2012), and permeability may be affected by solvents like DMSO (Kais et al., 2013).

One laboratory that routinely dechorionates embryos has an automated method for dechorionation and placement of embryos that avoids the technical limitations of traditional approaches to dechorionation (Mandrell et al., 2012) described above.

The IGG investigators agreed that use of dechorionated embryos is a key factor in experimental design with the potential to affect the outcomes and conclusions of exposure experiments. However, there are few systematic examinations of the utility of chorion removal in the literature. As previously mentioned, Panzica-Kelly (2015) reports slight increases in sensitivity with chorion removal and treatment. Henn and Braunbeck (2011) investigated removal of the chorion to improve the FET test. Their protocol used a static exposure method to test toxicity of Luviquat HM $552^{2}$, an aqueous solution of cationic polymers of approximately $400 \mathrm{kDa}$ in size, which are too large to readily

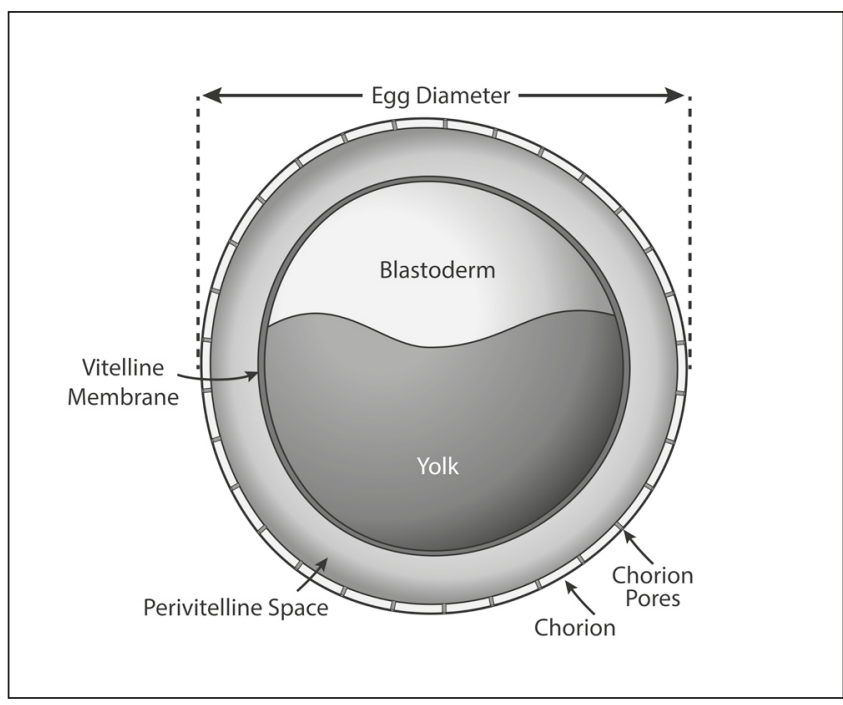

Fig. 5: General structure of the zebrafish egg

The zebrafish egg is approximately $0.7 \mathrm{~mm}$ in diameter. The chorion (outer layer) has a thickness of $3.5 \mu \mathrm{m}$ and is fenestrated with $0.5 \mu \mathrm{m}$ diameter pores allowing passage of water, ions, and chemicals. The fluid-filled perivitelline space overlays the vitelline membrane, which surrounds the yolk and the blastoderm, which will become the developing embryo. Figure adapted from Jones et al., 1978.

cross the chorion. The study found that dechorionation increased toxicity of these extremely large polymers. On the other hand, a consortium of biopharmaceutical companies that investigated the uptake of test chemicals found that, while there were chemicals with poor $(\leq 5 \%)$ embryo uptake, increasing the maximum test chemical concentration to $1000 \mu \mathrm{M}$ improved teratogen detection without requiring chorion removal (Ball et al., 2014).

A key finding from the IGG interviews and literature survey was that there are no studies examining the toxicity of a diverse chemical set in chorion-intact and dechorionated zebrafish embryos in which the same chemicals are compared simultaneously in multiple laboratories with accompanying uptake studies.

\section{Discussion}

The 2014 Collaborative Workshop on Aquatic Models and $21^{\text {st }}$ Century Toxicology (Planchart et al., 2016) highlighted the variability that currently exists among participating laboratories with respect to animal husbandry and protocols for chemical screening using zebrafish embryos. Previous efforts to evaluate different zebrafish methods have recommended harmonized protocols (Beekhuijzen et al., 2015). However, the SEAZIT team felt that given the diversity of experimental conditions in use for these studies, forcing researchers to use a single, unified protocol was

2 BASF (2011). Luviquat Technical Information Sheet. http://dewolfchem.com/wp-content/uploads/2013/08/Luviquat-TDS.pdf (accessed 01.05.2017). 
impractical and that the identification of key factors producing variability in assay outcomes was of the most use to researchers.

All of the IGG laboratories emphasized the importance of using genetically diverse fish stocks, implementing good husbandry practices with high-quality water and feed, maintaining consistent light/dark cycles for adult fish, and avoiding overbreeding of stock fish to obtain quality embryos. The laboratories also emphasized the importance of monitoring breeder fish fecundity, both to ensure availability of sufficient quantities of embryos for testing and as a general indicator of overall fish health.

IGG members described a diverse group of endpoints their laboratories utilized for the comprehensive interrogation of chemical toxicity in zebrafish. Among the IGG laboratories, there is variability in endpoints measured, the means of data capture and scoring, and the interpretation of phenotype alterations. Ducharme et al. (2013) recently surveyed zebrafish developmental toxicity studies and reported a high degree of variability in data collected in these studies. Zhang et al. (2017), using the data from Truong et al. (2014), recently reported an analysis method that develops weighting factors for endpoints, improves prediction of toxic effects, and provides evidence for developmental connections between highly correlated endpoints. Following on these findings, the SEAZIT project is attempting to define best practices for data capture, analysis, and reporting.

The IGG identified two specific parameters that can potentially influence study outcomes for chemical screening using zebrafish embryos: the presence versus absence of the chorion and the use of the static versus static-renewal exposure procedure. IGG members also agreed that, regardless of protocol design, a better understanding of chemical uptake in the zebrafish embryo model would greatly improve the utility of this model.

The input from the IGG members is currently being used to design an interlaboratory study to evaluate the effects of the chorion and exposure methods. Such a study is important to further define the role of protocol design elements in study outcome prior to any attempt to develop one or more harmonized protocols for zebrafish embryo tests. Comparisons of toxicity estimates across laboratories for a diverse chemical set with complimentary data on chemical uptake should allow the further refinement of best practices for a testing protocol(s), adoption of data standards, shared/common endpoints, use of ontologies and data mapping, and standards for data reporting that includes raw data. While the eventual development of a more defined zebrafish embryo test(s) for specified purposes would likely require the development of a well-defined protocol, the current effort focused on defining a set of parameters that all researchers should consider before they start to develop their assay and when publishing results. These advancements would greatly improve the ease with which zebrafish embryo screening data can be used to help inform a broad range of health-related research areas, including chemical hazard assessments. As the SEAZIT effort progresses, we continue to make connections with zebrafish researchers from the U.S. and overseas to collect a broad range of opinions and foster greater consistency in the experimental approaches employed.

\section{References}

Adatto, I., Lawrence, C., Thompson, M. and Zon, L. I. (2011). A new system for the rapid collection of large numbers of developmentally staged zebrafish embryos. PloS One 6, e21715. doi:10.1371/journal.pone.0021715

Aguirre-Martínez, G. V., Reinardy, H. C., Martín-Díaz, M. L. and Henry, T. B. (2017). Response of gene expression in zebrafish exposed to pharmaceutical mixtures: Implications for environmental risk. Ecotoxicol Environ Saf 142, 471-479. doi:10.1016/j.ecoenv.2017.04.038

Almond, K. M. and Trombetta, L. D. (2016). The effects of copper pyrithione, an antifouling agent, on developing zebrafish embryos. Ecotoxicology 25, 389-398. doi:10.1007/s10646015-1597-3

Armitage, J. M., Erickson, R. J., Luckenbach, T. et al. (2017). Assessing the bioaccumulation potential of ionizable organic compounds: Current knowledge and research priorities. Environ Toxicol Chem 36, 882-897. doi:10.1002/etc.3680

Attene-Ramos, M. S., Miller, N., Huang, R. et al. (2013). The Tox21 robotic platform for the assessment of environmental chemicals - From vision to reality. Drug Discov Today 18, 716-723. doi:10.1016/j.drudis.2013.05.015

Augustine-Rauch, K., Zhang, C. X. and Panzica-Kelly, J. M. (2010). In vitro developmental toxicology assays: A review of the state of the science of rodent and zebrafish whole embryo culture and embryonic stem cell assays. Birth Defects Res $C$ Embryo Today 90, 87-98. doi:10.1002/bdrc.20175

Augustine-Rauch, K., Zhang, C. X. and Panzica-Kelly, J. M. (2016). A developmental toxicology assay platform for screening teratogenic liability of pharmaceutical compounds. Birth Defects Res B Dev Reprod Toxicol 107, 4-20. doi:10.1002/ bdrb. 21168

Balik-Meisner, M., Truong, L., Scholl, E. H. et al. (2018a). Elucidating gene-by-environment interactions associated with differential susceptibility to chemical exposure. Environ Health Perspect 126, 067010. doi:10.1289/EHP2662

Balik-Meisner, M., Truong, L., Scholl, E. H. et al. (2018b). Population genetic diversity in zebrafish lines. Mamm Genome 29, 90-100. doi:10.1007/s00335-018-9735-x

Ball, J. S., Stedman, D. B., Hillegass, J. M. et al. (2014). Fishing for teratogens: A consortium effort for a harmonized zebrafish developmental toxicology assay. Toxicol Sci 139, 210-219. doi:10.1093/toxsci/kfu017

Barba-Escobedo, P. A. and Gould, G. G. (2012). Visual social preferences of lone zebrafish in a novel environment: Strain and anxiolytic effects. Genes Brain Behav 11, 366-373. doi:10.1111/j.1601-183X.2012.00770.x

Bartlett, D. H. and Silk, S. B. (2016). Office of laboratory animal welfare comments. Zebrafish 13, 563-564. doi:10.1089/ zeb.2016.1344

Beekhuijzen, M., de Koning, C., Flores-Guillén, M. E. et al. (2015). From cutting edge to guideline: A first step in harmonization of the zebrafish embryotoxicity test (ZET) by describing the most optimal test conditions and morphology scoring system. Reprod Toxicol 56, 64-76. doi:10.1016/j.reprotox.2015.06.050 
Berntssen, M. H., Julshamn, K. and Lundebye, A.-K. (2010). Chemical contaminants in aquafeeds and Atlantic salmon (Salmo salar) following the use of traditional- versus alternative feed ingredients. Chemosphere 78, 637-646. doi:10.1016/j. chemosphere.2009.12.021

Bonsignorio, D., Perego, L., Del Giacco, L. and Cotelli, F. (1996). Structure and macromolecular composition of the zebrafish egg chorion. Zygote 4, 101-108. doi:10.1017/ S0967199400002975

Braithwaite, V. A. and Boulcott, P. (2007). Pain perception, aversion and fear in fish. Dis Aquat Organ 75, 131-138. doi: 10.3354/dao075131

Brannen, K. C., Panzica-Kelly, J. M., Danberry, T. L. and Augustine-Rauch, K. A. (2010). Development of a zebrafish embryo teratogenicity assay and quantitative prediction model. Birth Defects Res B Dev Reprod Toxicol 89, 66-77. doi:10.1002/ bdrb. 20223

Braunbeck, T., Böttcher, M., Hollert, H. et al. (2005). Towards an alternative for the acute fish $\mathrm{LC}_{50}$ test in chemical assessment: The fish embryo toxicity test goes multi-species - An update. ALTEX 22, 87-102. doi:10.1016/j.aquatox.2009.12.008

Braunbeck, T., Kais, B., Lammer, E. et al. (2015). The fish embryo test (FET): Origin, applications, and future. Environ Sci Pollut Res Int 22, 16247-16261. doi:10.1007/s11356-0143814-7

Brown, K. H., Dobrinski, K. P., Lee, A. S. et al. (2012). Extensive genetic diversity and substructuring among zebrafish strains revealed through copy number variant analysis. Proc Natl Acad Sci U S A 109, 529-534. doi:10.1073/pnas.1112163109

Bugel, S. M., Tanguay, R. L. and Planchart, A. (2014). Zebrafish: A marvel of high-throughput biology for $21^{\text {st }}$ century toxicology. Curr Env Health Rep 1, 341-352. doi:10.1007/s40572014-0029-5

Burnison, B. K., Meinelt, T., Playle, R. et al. (2006). Cadmium accumulation in zebrafish (Danio rerio) eggs is modulated by dissolved organic matter (DOM). Aquat Toxicol 79, 185-191. doi:10.1016/j.aquatox.2006.06.010

Busquet, F., Nagel, R., von Landenberg, F. et al. (2008). Development of a new screening assay to identify proteratogenic substances using zebrafish Danio rerio embryo combined with an exogenous mammalian metabolic activation system (mDarT). Toxicol Sci 104, 177-188. doi:10.1093/toxsci/kfn065

Busquet, F., Strecker, R., Rawlings, J. M. et al. (2014). OECD validation study to assess intra- and inter-laboratory reproducibility of the zebrafish embryo toxicity test for acute aquatic toxicity testing. Regul Toxicol Pharmacol 69, 496-511. doi:10.1016/j.yrtph.2014.05.018

Cameron, I. L. and Hunter, K. E. (1984). Regulation of the permeability of the medaka fish embryo chorion by exogeneous sodium and calcium ions. J Exp Zool 231, 447-454. doi:10.1002/jez.1402310320

Ceol, C. J. and Houvras, Y. (2016). Uncharted waters: Zebrafish cancer models navigate a course for oncogene discovery. $A d v$ Exp Med Biol 916, 3-19. doi:10.1007/978-3-319-30654-4 1

Chatla, K., Gaunt, P. S., Petrie-Hanson, L. et al. (2016). Zebrafish sensitivity to botulinum neurotoxins. Toxins (Basel) 8, 132. doi: $10.3390 /$ toxins 8050132
Chen, J.-C. and Lru, P.-C. (1987). Accumulation of heavy metals in the nauplii of Artemia salina. JWAS 18, 84-93. doi:10.1111/j.1749-7345.1987.tb00422.x

Chen, T.-H., Wang, Y.-H. and Wu, Y.-H. (2011). Developmental exposures to ethanol or dimethylsulfoxide at low concentrations alter locomotor activity in larval zebrafish: Implications for behavioral toxicity bioassays. Aquat Toxicol 102, 162-166. doi:10.1016/j.aquatox.2011.01.010

Childs, B. and Der Kaloustian, V. M. (1968). Genetic heterogeneity. $N$ Engl J Med 279, 1205-1212. doi:10.1056/NEJM 196811282792206

Coe, T. S., Hamilton, P. B., Griffiths, A. M. et al. (2009). Genetic variation in strains of zebrafish (Danio rerio) and the implications for ecotoxicology studies. Ecotoxicology 18, 144-150. doi:10.1007/s10646-008-0267-0

Collins, F. S., Gray, G. M. and Bucher, J. R. (2008). Toxicology. Transforming environmental health protection. Science 319, 906-907. doi:10.1126/science.1154619

Collymore, C., Crim, M. J. and Lieggi, C. (2016). Recommendations for health monitoring and reporting for zebrafish research facilities. Zebrafish 13, Suppl 1, S138-148. doi:10.1089/zeb. 2015.1210

Cook, M. A., Johnson, R. B., Nicklason, P. et al. (2008). Marking live feeds with inert metal oxides for fish larvae feeding and nutrition studies. Aquacult Res 39, 347-353. doi:10.1111/ j.1365-2109.2007.01709.x

Creton, R.(2004). The calcium pump of the endoplasmic reticulum plays a role in midline signaling during early zebrafish development. Dev Brain Res 151, 33-41. doi:10.1016/j.devbrainres. 2004.03.016

Dai, Y. J., Jia, Y. F., Chen, N. et al. (2014). Zebrafish as a model system to study toxicology. Environ Toxicol Chem 33, 11-17. doi:10.1002/etc.2406

David, R. M., Jones, H. S., Panter, G. H. et al. (2012). Interference with xenobiotic metabolic activity by the commonly used vehicle solvents dimethylsulfoxide and methanol in zebrafish (Danio rerio) larvae but not Daphnia magna. Chemosphere 88, 912-917. doi:10.1016/j.chemosphere.2012.03.018

de Koning, C., Beekhuijzen, M., Tobor-Kapłon, M. et al. (2015). Visualizing compound distribution during zebrafish embryo development: The effects of lipophilicity and DMSO. Birth Defects Res B Dev Reprod Toxicol 104, 253-272. doi:10.1002/ bdrb. 21166

de Oliveira, G. A., de Lapuente, J., Teixido, E. et al. (2016). Textile dyes induce toxicity on zebrafish early life stages. Environ Toxicol Chem 35, 429-434. doi:10.1002/etc.3202

Dix, D. J., Houck, K. A., Martin, M. T. et al. (2007). The ToxCast program for prioritizing toxicity testing of environmental chemicals. Toxicol Sci 95, 5-12. doi:10.1093/toxsci/kfl103

Dodd, A., Curtis, P. M., Williams, L. C. and Love, D. R. (2000). Zebrafish: Bridging the gap between development and disease. Hum Mol Genet 9, 2443-2449. doi:10.1093/hmg/9.16.2443

Ducharme, N. A., Peterson, L. E., Benfenati, E. et al. (2013). Meta-analysis of toxicity and teratogenicity of 133 chemicals from zebrafish developmental toxicity studies. Reprod Toxicol 41, 98-108. doi:10.1016/j.reprotox.2013.06.070

Ducharme, N. A., Reif, D. M., Gustafsson, J. A. and Bondesson, 
M. (2015). Comparison of toxicity values across zebrafish early life stages and mammalian studies: Implications for chemical testing. Reprod Toxicol 55, 3-10. doi:10.1016/j.reprotox. 2014.09.005

Elkouby, Y. M. (2017). All in one - Integrating cell polarity, meiosis, mitosis and mechanical forces in early oocyte differentiation in vertebrates. Int J Dev Biol 61, 179-193. doi:10.1387/ ijdb.170030ye

Embry, M. R., Belanger, S. E., Braunbeck, T. A. et al. (2010). The fish embryo toxicity test as an animal alternative method in hazard and risk assessment and scientific research. Aquat Toxicol 97, 79-87. doi:10.1016/j.aquatox.2009.12.008

Erickson, R. J., McKim, J. M., Lien, G. J. et al. (2006). Uptake and elimination of ionizable organic chemicals at fish gills: I. Model formulation, parameterization, and behavior. Environ Toxicol Chem 25, 1512-1521. doi:10.1897/05-358R.1

Finn, R. N. (2007). The physiology and toxicology of salmonid eggs and larvae in relation to water quality criteria. Aquat Toxicol 81, 337-354. doi:10.1016/j.aquatox.2006.12.021

Forbes, E. L., Preston, C. D. and Lokman, P. M. (2010). Zebrafish (Danio rerio) and the egg size versus egg number trade off: Effects of ration size on fecundity are not mediated by orthologues of the Fec gene. Reprod Fertil Dev 22, 1015-1021. doi:10.1071/rd09257

Gao, Y., Zhang, G., Jelfs, B. et al. (2016). Computational classification of different wild-type zebrafish strains based on their variation in light-induced locomotor response. Comput Biol Med 69, 1-9. doi:10.1016/j.compbiomed.2015.11.012

Geisler, R., Rauch, G. J., Geiger-Rudolph, S. et al. (2007). Largescale mapping of mutations affecting zebrafish development. BMC Genomics 8, 11. doi:10.1186/1471-2164-8-11

Goldstone, J. V. and Stegeman, J. J. (2012). Methodological approaches to cytochrome P450 profiling in embryos. Methods Mol Biol 889, 265-275. doi:10.1007/978-1-61779-867-2_16

Gunnarsson, L., Jauhiainen, A., Kristiansson, E. et al. (2008). Evolutionary conservation of human drug targets in organisms used for environmental risk assessments. Environ Sci Technol 42, 5807-5813. doi:10.1021/es8005173

Gustafson, A. L., Stedman, D. B., Ball, J. et al. (2012). Inter-laboratory assessment of a harmonized zebrafish developmental toxicology assay - Progress report on phase I. Reprod Toxicol 33, 155-164. doi:10.1016/j.reprotox.2011.12.004

Haffter, P., Granato, M., Brand, M. et al. (1996). The identification of genes with unique and essential functions in the development of the zebrafish, Danio rerio. Development 123, 1-36. doi:10.1242/dev.153411

Hallare, A., Nagel, K., Köhler, H.-R. and Triebskorn, R. (2006). Comparative embryotoxicity and proteotoxicity of three carrier solvents to zebrafish (Danio rerio) embryos. Ecotoxicol Environ Saf 63, 378-388. doi:10.1016/j.ecoenv.2005.07.006

Henn, K. and Braunbeck, T. (2011). Dechorionation as a tool to improve the fish embryo toxicity test (FET) with the zebrafish (Danio rerio). Comp Biochem Physiol C Toxicol Pharmacol 153, 91-98. doi:10.1016/j.cbpc.2010.09.003

Hisaoka, K. K. (1958). Microscopic studies of the teleost chorion. Trans Am Microsc Soc 77, 240-243. doi:10.2307/3223685

Hisaoka, K. K. and Battle, H. I. (1958). The normal develop- mental stages of the zebrafish, Brachydanio rerio (Hamilton-Buchanan). J Morphol 102, 311-327. doi:10.1002/jmor. 1051020205

Hisaoka, K. K. and Firlit, C. F. (1960). Further studies on the embryonic development of the zebrafish, Brachydanio rerio (Hamilton-Buchanan). J Morphol 107, 205-225. doi:10.1002/ jmor. 1051070206

Horie, Y., Yamagishi, T., Takahashi, H. et al. (2017). Assessment of the lethal and sublethal effects of 20 environmental chemicals in zebrafish embryos and larvae by using OECD TG 212. J Appl Toxicol 37, 1245-1253. doi:10.1002/jat.3487

Howe, K., Clark, M. D., Torroja, C. F. et al. (2013). The zebrafish reference genome sequence and its relationship to the human genome. Nature 496, 498-503. doi:10.1038/nature12111

Janik, M., Kleinhans, F. W. and Hagedorn, M. (2000). Overcoming a permeability barrier by microinjecting cryoprotectants into zebrafish embryos (Brachydanio rerio). Cryobiology 41, 25-34. doi:10.1006/cryo.2000.2261

Johnson, S. L. and Zon, L. I. (1999). Genetic backgrounds and some standard stocks and strains used in zebrafish developmental biology and genetics. Methods Cell Biol 60, 357-359. doi:10.1016/S0091-679X(08)61910-X

Jones, P. W., Martin, D. M. and Hardy, J. D., Jr. (1978). Development of Fishes of the Mid-Atlantic Bight: An Atlas of Egg, Larval, and Juvenile Stages. Volume I: Acipenseride Through Ictaluridae. Washington, DC, USA: U.S. Fish and Wildlife Services. http://pubs.er.usgs.gov/publication/fwsobs78_12

Kais, B., Schneider, K. E., Keiter, S. et al. (2013). DMSO modifies the permeability of the zebrafish (Danio rerio) chorion-implications for the fish embryo test (FET). Aquat Toxicol 140-141, 229-238. doi:10.1016/j.aquatox.2013.05.022

Karlsen, O., van der Meeren, T., Rønnestad, I. et al. (2015). Copepods enhance nutritional status, growth and development in Atlantic cod (Gadus morhua L.) larvae - Can we identify the underlying factors? PeerJ 3, e902. doi:10.7717/peerj.902

Kim, K. T. and Tanguay, R. L. (2014). The role of chorion on toxicity of silver nanoparticles in the embryonic zebrafish assay. Environ Health Toxicol 29, e2014021. doi:10.5620/eht. e2014021

Kimmel, C. B., Ballard, W. W., Kimmel, S. R. et al. (1995). Stages of embryonic development of the zebrafish. Dev Dyn 203, 253-310. doi:10.1002/aja.1002030302

Kinth, P., Mahesh, G. and Panwar, Y. (2013). Mapping of zebrafish research: A global outlook. Zebrafish 10, 510-517. doi: 10.1089/zeb.2012.0854

Klüver, N., Ortmann, J., Paschke, H. et al. (2014). Transient overexpression of adh8a increases allyl alcohol toxicity in zebrafish embryos. PLoS One 9, e90619. doi:10.1371/journal. pone.0090619

Kurogi, K., Liu, T. A., Sakakibara, Y. et al. (2013). The use of zebrafish as a model system for investigating the role of the SULTs in the metabolism of endogenous compounds and xenobiotics. Drug Metab Rev 45, 431-440. doi:10.3109/036025 32.2013.835629

Lammer, E., Carr, G. J., Wendler, K. et al. (2009). Is the fish embryo toxicity test (FET) with the zebrafish (Danio rerio) a potential alternative for the fish acute toxicity test? 
Comp Biochem Physiol C Toxicol Pharmacol 149, 196-209. doi:10.1016/j.cbpc.2008.11.006

Lawrence, C. (2007). The husbandry of zebrafish (Danio rerio): A review. Aquaculture 269, 1-20. doi:10.1016/j.aquaculture. 2007.04.077

Lawrence, C. (2011). Advances in zebrafish husbandry and management. Methods Cell Biol 104, 429-451. doi:10.1016/ b978-0-12-374814-0.00023-9

Lawrence, C. (2012). The reproductive biology and spawning of zebrafish in laboratory settings. In P. McGrath (ed.), Zebrafish: Methods for Assessing Drug Safety and Toxicity (1-13). Hoboken, NJ, USA: John Wiley \& Sons, Inc. doi:10. 1002/9781118102138.ch1

Lawrence, C. and Mason, T. (2012). Zebrafish housing systems: A review of basic operating principles and considerations for design and functionality. ILAR J 53, 179-191. doi:10.1093/ ilar.53.2.179

Leet, J. K., Lindberg, C. D., Bassett, L. A. et al. (2014). High-content screening in zebrafish embryos identifies butafenacil as a potent inducer of anemia. PLoS One 9, e104190. doi:10.1371/ journal.pone.0104190

Lele, Z. and Krone, P. H. (1996). The zebrafish as a model system in developmental, toxicological and transgenic research. Biotechnol Adv 14, 57-72. doi:10.1016/0734-9750(96)00004-3

Lidster, K., Readman, G. D., Prescott, M. J. and Owen, S. F. (2017). International survey on the use and welfare of zebrafish Danio rerio in research. J Fish Biol 90, 1891-1905. doi:10. 1111/jfb. 13278

Lieschke, G. J. and Currie, P. D. (2007). Animal models of human disease: Zebrafish swim into view. Nat Rev Genet 8, 353367. doi:10.1038/nrg2091

Lu, J. X., Lin, T. T., Huang, Y. Q. et al. (2013). Nutrient enrichment effect of Antarctic krill (Euphausia superba) powder on the fatty acid and amino acid compositions in Artemia nauplii. Chin J Ecology 32, 1563-1570.

Mandrell, D., Truong, L., Jephson, C. et al. (2012). Automated zebrafish chorion removal and single embryo placement: Optimizing throughput of zebrafish developmental toxicity screens. J Lab Autom 17, 66-74. doi:10.1177/2211068211432197

Martins, J., Oliva Teles, L. and Vasconcelos, V. (2007). Assays with Daphnia magna and Danio rerio as alert systems in aquatic toxicology. Environ Int 33, 414-425. doi:10.1016/j. envint.2006.12.006

Matthews, J. L. (2004). Common diseases of laboratory zebrafish. Methods Cell Biol 77, 617-643. doi:10.1016/S0091679X(04)77033-8

Maule, A. G., Gannam, A. L. and Davis, J. W. (2007). Chemical contaminants in fish feeds used in federal salmonid hatcheries in the USA. Chemosphere 67, 1308-1315. doi:10.1016/j.chemosphere.2006.11.029

McCollum, C. W., Conde-Vancells, J., Hans, C. et al. (2016). Identification of vascular disruptor compounds by analysis in zebrafish embryos and mouse embryonic endothelial cells. Reprod Toxicol 70, 60-69. doi:10.1016/j.reprotox.2016.11.005

Meyer, B. M., Froehlich, J. M., Galt, N. J. and Biga, P. R. (2013). Inbred strains of zebrafish exhibit variation in growth performance and myostatin expression following fasting.
Comp Biochem Physiol, Part A Mol Integr Physiol 164, 1-9. doi:10.1016/j.cbpa.2012.10.004

Miller, G. W., Labut, E. M., Lebold, K. M. et al. (2012). Zebrafish (Danio rerio) fed vitamin E-deficient diets produce embryos with increased morphologic abnormalities and mortality. $J$ Nutr Biochem 23, 478-486. doi:10.1016/j.jnutbio.2011.02.002

Miller, G. W., Truong, L., Barton, C. L. et al. (2014). The influences of parental diet and vitamin $\mathrm{E}$ intake on the embryonic zebrafish transcriptome. Comp Biochem Physiol Part D Genomics Proteomics 10, 22-29. doi:10.1016/j.cbd.2014.02.001

Monroe, J. D., Manning, D. P., Uribe, P. M. et al. (2016). Hearing sensitivity differs between zebrafish lines used in auditory research. Hear Res 341, 220-231. doi:10.1016/j.heares. 2016.09.004

Murray, K. N., Dreska, M., Nasiadka, A. et al. (2011). Transmission, diagnosis, and recommendations for control of Pseudoloma neurophilia infections in laboratory zebrafish (Danio rerio) facilities. Comp Med 61, 322-329.

Nácher-Mestre, J., Serrano, R., Portolés, T. et al. (2014). Screening of pesticides and polycyclic aromatic hydrocarbons in feeds and fish tissues by gas chromatography coupled to high-resolution mass spectrometry using atmospheric pressure chemical ionization. J Agric Food Chem 62, 2165-2174. doi:10.1021/jf405366n

Nagel, R., Bresch, H., Caspers, N. et al. (1991). Effect of 3,4-dichloroaniline on the early life stages of the zebrafish (Brachydanio rerio): Results of a comparative laboratory study. Ecotoxicol Environ Saf 21, 157-164. doi:10.1016/01476513(91)90017-J

Nagel, R. (2002). DarT: The embryo test with the zebrafish Danio rerio - A general model in ecotoxicology and toxicology. ALTEX 19, Suppl 1, 38-48. http://www.altex.ch/resources/ altex_2002_Supp_1_38_48_Nagel.pdf

Nash, C. E. (1973). Automated mass-production of Artemia salina nauplii for hatcheries. Aquaculture 2, 289-298. doi:10.1016/0044-8486(73)90161-0

Ninness, M. M., Stevens, E. D. and Wright, P. A. (2006). Removal of the chorion before hatching results in increased movement and accelerated growth in rainbow trout (Oncorhynchus mykiss) embryos. J Exp Biol 209, 1874-1882. doi:10.1242/ jeb.02200

NRC (2007). Toxicity Testing in the 21 ${ }^{\text {st }}$ Century: $A$ vision and a Strategy. Washington, DC, USA: National Academies Press. http://books.nap.edu/openbook.php?record_id=11970 (accessed 17.09.2009).

NRC (2017). Using $21^{\text {st }}$ Century Science to Improve Risk-Related Evaluations. Washington, DC, USA: National Academies Press. doi:10.17226/24635

Nüsslein-Volhard, C. and Dahm, R. (2002). Zebrafish: A Practical Approach. New York, USA: Oxford University Press. https://books.google.com/books?id=jfm0QgAACAAJ

OECD - Organisation for Economic Co-operation and Development (1992). Test No. 203: Fish, Acute Toxicity Test. OECD Guidelines for the Testing of Chemicals, Section 2: Biotic Effects. Paris, France: OECD Publishing. doi:10.1787/20745761 OECD (1998). Test No. 212: Fish, Short-term Toxicity Test on Embryo and Sac-Fry Stages. OECD Guidelines for the Testing 
of Chemicals, Section 2: Biotic Effects. Paris, France: OECD Publishing. http://www.oecd-ilibrary.org/environment/test-no212-fish-short-term-toxicity-test-on-embryo-and-sac-frystages_9789264070141-en (accessed 27.12.2016).

OECD (2002). OECD Series on Testing and Assessment Number 23: Guidance Document on Aquatic Toxicity Testing of Difficult Substances and Mixtures. Paris, France: OECD Publishing. doi:10.1787/9789264078406-en

OECD (2013a). Test No. 210: Fish, early-life stage toxicity test. OECD Guidelines for the Testing of Chemicals, Section 2: Biotic Effects. Paris, France: OECD Publishing. http://www. oecd-ilibrary.org/content/book/9789264203785-en (accessed 02.05.2017).

OECD (2013b). Test No. 236: Fish Embryo Acute Toxicity (FET) Test. OECD Guidelines for the Testing of Chemicals, Section 2: Biotic Effects. Paris, France: OECD Publishing. http://www.oecd-ilibrary.org/environment/test-no-236-fishembryo-acute-toxicity-fet-test_9789264203709-en

Otte, J. C., Schmidt, A. D., Hollert, H. and Braunbeck, T. (2010). Spatio-temporal development of CYP1 activity in early life-stages of zebrafish (Danio rerio). Aquat Toxicol 100, 3850. doi:10.1016/j.aquatox.2010.07.006

Padilla, S., Corum, D., Padnos, B. et al. (2012a). Zebrafish developmental screening of the ToxCast Phase I chemical library. Reprod Toxicol 33, 174-187. doi:10.1016/j.reprotox. 2011.10.018

Padilla, S., Corum, D., Padnos, B. et al. (2012b). Zebrafish developmental screening of the ToxCast ${ }^{\mathrm{TM}}$ Phase I chemical library. Reprod Toxicol 33, 174-187. doi:10.1016/j.reprotox. 2011.10.018

Panzica-Kelly, J. M., Zhang, C. X., Danberry, T. L. et al. (2010). Morphological score assignment guidelines for the dechorionated zebrafish teratogenicity assay. Birth Defects Res B Dev Reprod Toxicol 89, 382-395. doi:10.1002/bdrb.20260

Panzica-Kelly, J. M., Zhang, C. X. and Augustine-Rauch, K. A. (2015). Optimization and performance assessment of the chorion-off [dechorinated] zebrafish developmental toxicity assay. Toxicol Sci 146, 127-134. doi:10.1093/toxsci/kfv076

Pardo-Martin, C., Chang, T. Y., Koo, B. K. et al. (2010). High-throughput in vivo vertebrate screening. Nat Methods 7 , 634-636. doi:10.1038/nmeth.1481

Perkins, E. J., Ankley, G. T., Crofton, K. M. et al. (2013). Current perspectives on the use of alternative species in human health and ecological hazard assessments. Environ Health Perspect 121, 1002-1010. doi:10.1289/ehp.1306638

Perry, S. F., Wilson, R. J., Straus, C. et al. (2001). Which came first, the lung or the breath? Comp Biochem Physiol A Mol Integr Physiol 129, 37-47.

Planchart, A., Mattingly, C. J., Allen, D. et al. (2016). Advancing toxicology research using in vivo high throughput toxicology with small fish models. ALTEX 33, 435-452. doi:10.14573/ altex.1601281

Postlethwait, J. H., Woods, I. G., Ngo-Hazelett, P. et al. (2000). Zebrafish comparative genomics and the origins of vertebrate chromosomes. Genome Res 10, 1890-1902. doi:10.1101/gr. 164800

Quadros, V. A., Silveira, A., Giuliani, G. S. et al. (2016). Strain- and context-dependent behavioural responses of acute alarm substance exposure in zebrafish. Behav Processes 122, 1-11. doi:10.1016/j.beproc.2015.10.014

Raftery, T. D., Isales, G. M., Yozzo, K. L. and Volz, D. C. (2014). High-content screening assay for identification of chemicals impacting spontaneous activity in zebrafish embryos. Environ Sci Technol 48, 804-810. doi:10.1021/es404322p

Rawson, D. M., Zhang, T., Kalicharan, D. and Jongebloed, W. L. (2000). Field emission scanning electron microscopy and transmission electron microscopy studies of the chorion, plasma membrane and syncytial layers of the gastrula-stage embryo of the zebrafish Brachydanio rerio: A consideration of the structural and functional relationships with respect to cryoprotectant penetration. Aquacult Res 31, 325-336. doi:10.1046/j.1365-2109.2000.00401.x

Rovida, C. and Hartung, T. (2009). Re-evaluation of animal numbers and costs for in vivo tests to accomplish REACH legislation requirements for chemicals - A report by the transatlantic think tank for toxicology $\left(\mathrm{t}^{4}\right)$. ALTEX 26, 187-208. doi:10.14573/altex.2009.3.187

Russell, W. M. S. and Burch, R. L. (1992). The Principles of Humane Experimental Technique. 14 ${ }^{\text {th }}$ edition. Potters Bar, Herts, UK: Universities Federation for Animal Welfare.

Scheil, V., Kienle, C., Osterauer, R. et al. (2009). Effects of 3,4-dichloroaniline and diazinon on different biological organisation levels of zebrafish (Danio rerio) embryos and larvae. Ecotoxicology 18, 355-363. doi:10.1007/s10646-008-0291-0

Schulte, C. (1994). Testing acute toxicity in the embryo of zebrafish, Brachydanio rerio, as an alternative to the acute fish test: Preliminary results. Altern Lab Anim 22, 12-19.

Seale, A. (1933). The brine shrimp (Artemia) as a satisfactory live food for fishes. Transactions of the American Fisheries Society 63, 129-130. doi:10.1577/1548-8659(1933)63[129 :TBSAAA]2.0.CO;2

Seguret, A., Collignon, B. and Halloy, J. (2016). Strain differences in the collective behaviour of zebrafish (Danio rerio) in heterogeneous environment. $R$ Soc Open Sci 3, 160451. doi:10.1098/rsos.160451

Sneddon, L. U. (2009). Pain perception in fish: Indicators and endpoints. ILAR J 50, 338-342. doi:10.1093/ilar.50.4.338

Sneddon, L. U., Halsey, L. G. and Bury, N. R. (2017). Considering aspects of the 3 Rs principles within experimental animal biology. J Exp Biol 220, 3007-3016. doi:10.1242/jeb.147058

Solnica-Krezel, L., Stemple, D. L. and Driever, W. (1995). Transparent things: Cell fates and cell movements during early embryogenesis of zebrafish. Bioessays 17, 931-939. doi:10.1002/bies.950171106

Spence, R. and Smith, C. (2005). Male territoriality mediates density and sex ratio effects on oviposition in the zebrafish, Danio rerio. Anim Behav 69, 1317-1323. doi:10.1016/j.anbehav.2004.10.010

Spence, R. (2011). Zebrafish ecology and behaviour. Neuromethods 52, 1-46. doi:10.1007/978-1-60761-922-2_1

Stanton, M. F. (1965). Diethylnitrosamine-induced hepatic degeneratoin and neoplasia in the aquarium fish, Brachydanio rerio. J Natl Cancer Inst 34, 117-130. doi:10.1093/jnci/34.1.117 Strahle, U., Scholz, S., Geisler, R. et al. (2012). Zebrafish em- 
bryos as an alternative to animal experiments - A commentary on the definition of the onset of protected life stages in animal welfare regulations. Reprod Toxicol 33, 128-132. doi:10.1016/j.reprotox.2011.06.121

Streisinger, G., Walker, C., Dower, N. et al. (1981). Production of clones of homozygous diploid zebra fish (Brachydanio rerio). Nature 291, 293-296. doi:10.1038/291293a0

Streisinger, G. (1984). Attainment of minimal biological variability and measurements of genotoxicity: Production of homozygous diploid zebra fish. Natl Cancer Inst Monogr 65, 53-58.

Tannenbaum, J. and Bennett, B. T. (2015). Russell and Burch's 3Rs then and now: The need for clarity in definition and purpose. J Am Assoc Lab Anim Sci 54, 120-132.

Tao, T. and Peng, J. (2009). Liver development in zebrafish (Danio rerio). J Genet Genomics 36, 325-334. doi:10.1016/ S1673-8527(08)60121-6

Thomas, L. T., Welsh, L., Galvez, F. and Svoboda, K. R. (2009). Acute nicotine exposure and modulation of a spinal motor circuit in embryonic zebrafish. Toxicol Appl Pharmacol 239, 1-12. doi:10.1016/j.taap.2008.08.023

Tice, R. R., Austin, C. P., Kavlock, R. J. and Bucher, J. R. (2013). Improving the human hazard characterization of chemicals: A Tox21 update. Environ Health Perspect 121, 756-765. doi:10.1289/ehp.1205784

Truong, L., Reif, D. M., St Mary, L. et al. (2014). Multidimensional in vivo hazard assessment using zebrafish. Toxicol Sci 137, 212-233. doi:10.1093/toxsci/kft235

Truong, L., Simonich, M. T. and Tanguay, R. L. (2016). Better, faster, cheaper: Getting the most out of high-throughput screening with zebrafish. Methods Mol Biol 1473, 89-98. doi:10.1007/978-1-4939-6346-1_10

van den Bos, R., Mes, W., Galligani, P. et al. (2017). Further characterisation of differences between TL and AB zebrafish (Danio rerio): Gene expression, physiology and behaviour at day 5 of the larval stage. PLoS One 12, e0175420. doi:10.1371/ journal.pone.0175420

Van Slyke, C. E., Bradford, Y. M., Westerfield, M. and Haendel, M. A. (2014). The zebrafish anatomy and stage ontologies: Representing the anatomy and development of Danio rerio. $J$ Biomed Semantics 5, 12. doi:10.1186/2041-1480-5-12

Varga, Z. M. (2011). Aquaculture and husbandry at the zebrafish international resource center. Methods Cell Biol 104, 453-478. doi:10.1016/B978-0-12-374814-0.00024-0

Varshney, G. K., Sood, R. and Burgess, S. M. (2015). Understanding and editing the zebrafish genome. Adv Genet 92, 1-52. doi:10.1016/bs.adgen.2015.09.002

Vignet, C., Bégout, M. L., Péan, S. et al. (2013). Systematic screening of behavioral responses in two zebrafish strains. Zebrafish 10, 365-375. doi:10.1089/zeb.2013.0871

Vikas, P. A., Chakraborty, K., Sajeshkumar, N. K. et al. (2012).
Unraveling the effects of live microalgal enrichment on Artemia nauplii. Indian J Fisheries 59, 111-121.

Vliet, S. M., Ho, T. C. and Volz, D. C. (2017). Behavioral screening of the LOPAC(1280) library in zebrafish embryos. Toxicol Appl Pharmacol 329, 241-248. doi:10.1016/j. taap.2017.06.011.

Weigt, S., Huebler, N., Braunbeck, T. et al. (2010). Zebrafish teratogenicity test with metabolic activation (mDarT): Effects of phase I activation of acetaminophen on zebrafish Danio rerio embryos. Toxicology 275, 36-49. doi:10.1016/j. tox.2010.05.012

Weigt, S., Huebler, N., Strecker, R. et al. (2011). Zebrafish (Danio rerio) embryos as a model for testing proteratogens. Toxicology 281, 25-36. doi:10.1016/j.tox.2011.01.004

Westerfield, M. (2000). The Zebrafish Book: A Guide for the Laboratory Use of Zebrafish Danio (Brachydanio) rerio. $4^{\text {th }}$ edition. Eugene, Oregon, USA: University of Oregon Press. http://zfin.org/zf_info/zfbook/zfbk.html

Whipps, C. M., Lieggi, C. and Wagner, R. (2012). Mycobacteriosis in zebrafish colonies. ILAR J 53, 95-105. doi:10.1093/ ilar.53.2.95

White, R. M. (2015). Cross-species oncogenomics using zebrafish models of cancer. Curr Opin Genet Dev 30, 73-79. doi:10.1016/j.gde.2015.04.006

Wiegand, C., Pflugmacher, S., Giese, M. et al. (2000). Uptake, toxicity, and effects on detoxication enzymes of atrazine and trifluoroacetate in embryos of zebrafish. Ecotoxicol Environ Saf 45, 122-131. doi:10.1006/eesa.1999.1845

Yozzo, K. L., Isales, G. M., Raftery, T. D. and Volz, D. C. (2013). High-content screening assay for identification of chemicals impacting cardiovascular function in zebrafish embryos. Environ Sci Technol 47, 11302-11310. doi:10.1021/es403360y

Zhang, G., Roell, K. R., Truong, L. et al. (2017). A data-driven weighting scheme for multivariate phenotypic endpoints recapitulates zebrafish developmental cascades. Toxicol Appl Pharmacol 314, 109-117. doi:10.1016/j.taap.2016.11.010

Zuberi, A. and Lutz, C. (2016). Mouse models for drug discovery. Can new tools and technology improve translational power? ILAR J 57, 178-185. doi:10.1093/ilar/ilw021

\section{Conflict of interest}

The authors declare that they have no competing financial interests.

\section{Acknowledgments}

This project was funded in whole or in part with Federal funds from the intramural research program of the National Institute of Environmental Health Sciences, National Institutes of Health under Contract No. HHSN273201500010C. 